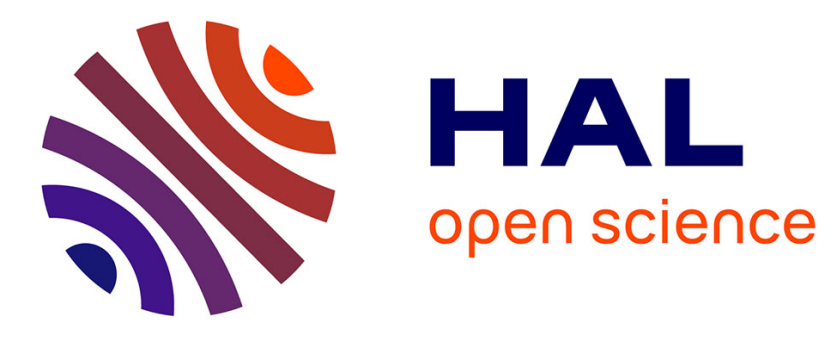

\title{
Intricate Axioms as Interaction Axioms
}

Guillaume Aucher

\section{- To cite this version:}

Guillaume Aucher. Intricate Axioms as Interaction Axioms. Studia Logica, 2015, pp.28. 10.1007/s11225-015-9609-0 . hal-01193284

\section{HAL Id: hal-01193284 \\ https://inria.hal.science/hal-01193284}

Submitted on 4 Sep 2015

HAL is a multi-disciplinary open access archive for the deposit and dissemination of scientific research documents, whether they are published or not. The documents may come from teaching and research institutions in France or abroad, or from public or private research centers.
L'archive ouverte pluridisciplinaire HAL, est destinée au dépôt et à la diffusion de documents scientifiques de niveau recherche, publiés ou non, émanant des établissements d'enseignement et de recherche français ou étrangers, des laboratoires publics ou privés. 


\title{
Intricate Axioms as Interaction Axioms*
}

\author{
Guillaume Aucher ${ }^{\dagger}$ \\ University of Rennes 1 \\ INRIA \\ 263, Avenue du Général Leclerc \\ 35042 Rennes Cedex, France
}

\begin{abstract}
In epistemic logic, some axioms dealing with the notion of knowledge are rather convoluted and difficult to interpret intuitively, even though some of them, such as the axioms .2 and .3, are considered to be key axioms by some epistemic logicians. We show that they can be characterized in terms of understandable interaction axioms relating knowledge and belief or knowledge and conditional belief. In order to show it, we first sketch a theory dealing with the characterization of axioms in terms of interaction axioms in modal logic. We then apply the main results and methods of this theory to obtain specific results related to epistemic and doxastic logics.
\end{abstract}

Keywords: Modal Logic, Epistemic logic, Interaction Axiom, Definability of Modalities.

\section{Introduction}

One of the goals of modern epistemic logic is to elucidate the nature of the interaction between knowledge and belief by means of formal and logical methods. On the basis of a semantics very close to the Kripke semantics of modal logic, Hintikka [18] and subsequent philosophers and logicians tried to formulate explicit principles governing and relating expressions of the form " $a$ knows that $\varphi$ " (subsequently formalized as $K \varphi$ ) and " $a$ believes that $\varphi$ " (subsequently formalized as $B \varphi$ ), where $a$ is a human agent and $\varphi$ is a proposition. In other words, they sought to determine 'the' logic of knowledge and belief, or at least of idealized versions of these notions. Their quest was grounded in the observation that our intuitions about these epistemic notions comply with some systematic reasoning properties, and was driven by the attempt to better understand and elucidate them [24, p. 15]. For example, the interaction axioms $K \varphi \rightarrow B \varphi$ and

\footnotetext{
${ }^{*}$ A short version of this article appears in [2]. This short version only deals with axioms .2 and .4 and does not deal with conditional beliefs.

${ }^{\dagger}$ Email: guillaume.aucher@irisa.fr
} 
$B \varphi \rightarrow K B \varphi$ are often considered to be intuitive principles: if agent $a$ knows $\varphi$ then (s)he also believes $\varphi$, and if agent $a$ believes $\varphi$, then (s)he knows that (s)he believes $\varphi$. As a matter of fact, assessing whether a given principle holds true or not raises our own awareness of these epistemic notions and reveals to us some of their essential properties.

In computer science, the logic of knowledge is usually considered to be S5, which is obtained by adding to the minimal normal modal logic $\mathrm{K}$ the axioms $K \varphi \rightarrow \varphi(\mathrm{T}), K \varphi \rightarrow K K \varphi$ (4) and $\neg K \varphi \rightarrow K \neg K \varphi$ (5). This last axiom 5 is falsified in a situation where the agent has mistaken beliefs. For this very reason, it has been attacked by various philosophers because it cannot hold in general: agents sometimes have mistaken beliefs. ${ }^{1}$ Dropping this axiom 5 from S5, we obtain the logic S4. Between the logics S4 and S5, a rich variety of weaker logics of knowledge have been proposed and examined by epistemic logicians [25], such as S4.2, S4.3 and S4.4. Even if these logics are characterized by axioms which are rather intricate, some of them have been proclaimed by some epistemic logicians as key axioms characterizing the notion of knowledge. For example, Lenzen claimed that "[t]here is strong evidence in favor of the assumption that S4.2 is the logic of knowledge" [25, p. 33], where the axiom .2 is $\neg K \neg K \varphi \rightarrow K \neg K \neg \varphi$. Likewise, Kutschera argues for S4.4 as the logic of knowledge, where the axiom .4 is $(\varphi \wedge \neg K \neg K \varphi) \rightarrow K \varphi$ [21]. As one can easily observe, it is difficult to provide these axioms with a natural and easily understandable reading. In fact, Lenzen derived his axiom .2 from a set of interaction axioms relating knowledge and belief (viewed as some sort of conviction). Similarly, the logic S4.3 is the logic S4 to which is added the axiom .3: $\neg K \neg \varphi \wedge \neg K \neg \psi \rightarrow \neg K \neg(\varphi \wedge \neg K \neg \psi) \vee$ $\neg K \neg(\varphi \wedge \psi) \vee \neg K \neg(\psi \wedge \neg K \neg \varphi)$. Again, as one can easily notice, it is difficult to provide this axiom with a natural and easily understandable reading. Stalnaker argues that a certain "defeasibility theory" of knowledge gives S4.3 [32, p.190].

To better grasp the intuitions underlying these intricate axioms .2, .3 and .4 , we show that they can be characterized equivalently in terms of interaction axioms relating knowledge and belief or knowledge and conditional belief. In order to do so, we first need to explain what we mean by "interaction axiom" and what we mean by "characterizing an axiom in terms of interaction axioms". This will lead us to develop a basic theory in modal logic dealing with these notions. Then, we will apply the general results of this theory to the specific case of epistemic logic. Note that such a theory has never been developed in the modal logic literature, neither in the context of multi-modal logics nor in the context of combinations of modal logics such as products and fusion [26, 14].

This article is divided in two parts: a "theoretical" part (Section 2) which presents our basic theory dealing with the characterization of axioms in terms of

\footnotetext{
${ }^{1}$ For example, assume that a university lecturer believes (is certain) that one of her colleague's seminars is on Thursday (formally $B p$ ). She is actually wrong because it is on Tuesday $(\neg p)$. Therefore, she does not know that her colleague's seminar is on Tuesday $(\neg K p)$. If we assume that axiom 5 is valid then we should conclude that she knows that she does not know that her colleague's seminar is on Tuesday $(K \neg K p)$ (and therefore she also believes that she does not know it: $B \neg K p$ ). This is obviously counterintuitive. More generally, axiom 5 is invalidated when the agent has mistaken beliefs which can be due for example to misperceptions, lies or other forms of deception.
} 
interaction axioms, and an "applicative" part (Sections 3, 4, 5, 6) where we apply some of the results of Section 2 to epistemic and doxastic logics. These logics are recalled in Section 3. The interaction axioms introduced in the literature are discussed in Section 4 and our main results related to epistemic logic are in Sections 5 and 6 .

Note. The missing proofs of propositions and theorems can be found in the appendix.

\section{Towards a Theory of Interaction Axioms}

In this section, we start by recalling the basics of modal logic (Section 2.1). Then, we present our basic meta-theory of modal logic dealing with interaction axioms. Firstly, we define what we mean by "interaction axiom" and what we mean by "characterizing an axiom in terms of interaction axiom" (Section 2.2). Secondly, we give general results which provide some necessary and sufficient conditions for an axiom to be characterized by a set of interaction axioms when one of the modalities is defined in terms of the other modality (Section 2.3).

\section{$2.1 \quad$ Modal Logic}

In this subsection, we recall the basics of modal logic. The semantics we consider for modal logic is the standard Kripke semantics.

\subsubsection{Syntax}

In the rest of the article, $\mathbb{P}$ is a set of propositional letters and $\mathbb{A} \subseteq\{1,2\}$, with $\mathbb{P}$ and $\mathbb{A}$ non-empty. We define the modal language $\mathcal{L}_{\mathbb{A}}$ by the following BNF grammar:

$$
\mathcal{L}_{\mathbb{A}}: \varphi \quad:=p|\neg \varphi|(\varphi \wedge \varphi) \mid[i] \varphi
$$

where $p$ ranges over $\mathbb{P}$ and $i$ ranges over $\mathbb{A}$. The formula $T$ is an abbreviation for $p \vee \neg p$ (for some fixed $p \in \mathbb{P}$ ), the formula $\perp$ is an abbreviation for $\neg \top$, $\varphi \vee \psi$ an abbreviation for $\neg(\neg \varphi \wedge \neg \psi), \varphi \rightarrow \psi$ an abbreviation for $\neg \varphi \vee \psi$, and $\langle i\rangle \varphi$ an abbreviation for $\neg[i] \neg \varphi$. An occurrence of a proposition letter $p$ is a positive occurrence if it is in the scope of an even number of negation signs ᄀ. A formula $\varphi$ is positive in $p$ if all occurrences of $p$ in $\varphi$ are positive. If $\Gamma:=\left\{\varphi_{1}, \ldots, \varphi_{n}\right\} \subseteq \mathcal{L}_{\mathbb{A}}$, then $\bigwedge \Gamma$ is an abbreviation for $\varphi_{1} \wedge \ldots \wedge \varphi_{n}$.

A (modal) $\operatorname{logic} \mathrm{L}$ for the modal language $\mathcal{L}_{\mathbb{A}}$ is a set of formulas of $\mathcal{L}_{\mathbb{A}}$ called theorems which contains all propositional tautologies and which is closed under modus ponens, that is, if $\varphi \in \mathrm{L}$ and $\varphi \rightarrow \psi \in \mathrm{L}$, then $\psi \in \mathrm{L}$, and closed under uniform substitution, that is, if $\varphi$ belongs to $L$ then so do all of its substitution instances (see [7, Def. 1.18] for the definition of a substitution instance).

A modal logic is usually defined by a set of inference rules and of formulas called axioms. A formula is a theorem of the modal logic if it can be derived 
by successively applying (some of) the inference rules to (some of) the axioms. We are interested here in normal modal logics. These modal logics contain the axiom schema $([i](\varphi \rightarrow \psi) \wedge[i] \varphi) \rightarrow[i] \psi$, and the inference rule of necessitation: from $\varphi \in \mathrm{L}$, infer $[i] \varphi \in \mathrm{L}$, for all $i \in \mathbb{A}$. Let $A \subseteq \mathcal{L}_{\mathbb{A}}$. A modal logic for $\mathcal{L}_{\mathbb{A}}$ generated by the set $A$ is the smallest normal modal logic for $\mathcal{L}_{\mathbb{A}}$ containing $A$. In that case, the formulas of $A$ are called axioms. The smallest of all normal modal logics for $\mathcal{L}_{\mathbb{A}}$ is denoted $\mathrm{K}$.

If $L$ and $L^{\prime}$ are two sets of formulas of $\mathcal{L}_{\mathbb{A}}$ (possibly logics), we denote by $\mathrm{L}+\mathrm{L}^{\prime}$ the modal logic for $\mathcal{L}_{\mathbb{A}}$ generated by $\mathrm{L} \cup \mathrm{L}^{\prime}$ (it is very similar to the fusion of $L$ and $L^{\prime}[26,14]$ ). If $x$ is a formula of $\mathcal{L}_{\mathbb{A}}$, then $L+x$ abusively denotes $L+\{x\}$. Note that $L+L^{\prime}$ may be different from $L \cup L^{\prime}$ in general, because $L \cup L^{\prime}$ may not be closed under modus ponens or uniform substitution.

Let $\mathrm{x} \in \mathcal{L}_{\mathbb{A}}$ and let $X, X^{\prime} \subseteq \mathcal{L}_{\mathbb{A}}$. We say that $\mathrm{x}$ is derivable from $X$ in $L$ when $\mathrm{x} \in \mathrm{L}+X$ and in that case we write $X \vdash_{\mathrm{L}} \mathrm{x}$. We also write $X \vdash_{\mathrm{L}} X^{\prime}$ when $X \vdash_{\mathrm{L}} \mathrm{x}^{\prime}$ for all $\mathrm{x}^{\prime} \in X^{\prime}$, and $X>_{\mathrm{L}} X^{\prime}$ when it holds that $X \vdash_{\mathrm{L}} X^{\prime}$ but it does not hold that $X^{\prime} \vdash_{\mathrm{L}} X$.

\subsubsection{Kripke Semantics.}

The Kripke semantics will be used only in the proof of Theorem 1. A (bi-modal) Kripke model $\mathcal{M}$ is a tuple $\mathcal{M}=\left(W, R_{1}, R_{2}, V\right)$ where $W$ is a non-empty set of possible worlds, $R_{1}, R_{2} \in 2^{W \times W}$ are binary relations over $W$ called accessibility relations, and $V: \mathbb{P} \rightarrow 2^{W}$ is called a valuation and assigns to each propositional letter $p \in \mathbb{P}$ a subset of $W$. A Kripke frame $\mathcal{F}$ is a Kripke model without a valuation. We often denote by $R_{i}(w)$ the set $R_{i}(w):=\left\{v \in W \mid w R_{i} v\right\}$ and we abusively write $w \in \mathcal{M}$ when $w \in W$. In that case, $(\mathcal{M}, w)$ is called a pointed Kripke model and we denote by $\mathcal{K}$ the set of all pointed Kripke models.

Let $\varphi \in \mathcal{L}_{\mathbb{A}}$, let $\mathcal{M}$ be a Kripke model and let $w \in \mathcal{M}$. The satisfaction relation $\mathcal{M}, w \models \varphi$ is defined inductively as follows:

$$
\begin{array}{lll}
\mathcal{M}, w \models p & \text { iff } & w \in V(p) \\
\mathcal{M}, w=\varphi \wedge \varphi^{\prime} & \text { iff } & \mathcal{M}, w \models \varphi \text { and } \mathcal{M}, w \models \varphi^{\prime} \\
\mathcal{M}, w=\neg \varphi & \text { iff } & \text { not } \mathcal{M}, w \models \varphi \\
\mathcal{M}, w \models[i] \varphi & \text { iff } & \text { for all } v \in R_{i}(w), \mathcal{M}, v \models \varphi
\end{array}
$$

If $\Gamma \subseteq \mathcal{L}_{\mathbb{A}}$, then we write $\mathcal{M}, w \models \Gamma$ when $\mathcal{M}, w \models \varphi$ for all $\varphi \in \Gamma$. Likewise, if $\mathcal{F}=\left(W, R_{1}, R_{2}\right)$ is an epistemic-doxastic frame, then we abusively write $w \in \mathcal{F}$ for $w \in W$. We also write $\Gamma \models \varphi$ when for all $(\mathcal{M}, w) \in \mathcal{K}, \mathcal{M}, w \models \Gamma$ implies that $\mathcal{M}, w=\varphi$. If $\Gamma$ is a set of formulas of $\mathcal{L}_{1,2}$, then we write $\mathcal{F} \models \Gamma$ when for all $\varphi \in \Gamma$ and all valuation $V,(\mathcal{F}, V) \models \varphi$, and we say that $\Gamma$ is valid in $\mathcal{F}$.

\subsection{Interaction Axioms}

In the sequel, $L_{1}$ and $L_{2}$ are two normal modal logics for $\mathcal{L}_{1}$ and $\mathcal{L}_{2}$ respectively, and $L_{1,2}$ is a normal modal logic for $\mathcal{L}_{1,2}$ (we abusively write $\mathcal{L}_{1,2}$ for $\mathcal{L}_{\{1,2\}}$ and $\mathcal{L}_{i}$ for $\mathcal{L}_{\{i\}}$, where $\left.i \in\{1,2\}\right)$. Intuitively, an interaction axiom is a formula 
which cannot be equivalent to any formula of the modal language with a single modality.

Definition 1. A set of interaction axioms w.r.t. a pair of $\operatorname{logics}\left(\mathrm{L}_{1}, \mathrm{~L}_{2}\right)$ is a finite set of formulas $\Gamma \subseteq \mathcal{L}_{1,2}$ for which there is no $\chi \in \mathcal{L}_{1} \cup \mathcal{L}_{2}$ such that

$$
\chi \leftrightarrow \bigwedge \Gamma \in \mathrm{L}_{1}+\mathrm{L}_{2} .
$$

In the sequel, $\mathrm{x}$ is a formula of $\mathcal{L}_{1}$ and $\Gamma$ is a set of interaction axioms w.r.t. $\left(\mathrm{L}_{1}, \mathrm{~L}_{2}\right)$.

Definition 2. We say that $x$ is characterized by the set of interaction axioms $\Gamma$ w.r.t. $\left(\mathrm{L}_{1}, \mathrm{~L}_{2}\right)$ when

$$
\mathrm{L}_{1}+\mathrm{x}=\left(\mathrm{L}_{1}+\mathrm{L}_{2}+\Gamma\right) \cap \mathcal{L}_{1} .
$$

Moreover, $\mathrm{x}$ is conservatively characterized by $\Gamma$ w.r.t. $\left(\mathrm{L}_{1}, \mathrm{~L}_{2}\right)$ when the set of interaction axioms $\Gamma$ satisfies the following condition as well:

$$
\mathrm{L}_{2}=\left(\mathrm{L}_{1}+\mathrm{L}_{2}+\Gamma\right) \cap \mathcal{L}_{2}
$$

Intuitively, Definition 2 tells us that an axiom $\mathrm{x}$ is characterized by a set of interaction axioms $\Gamma$ if, when we add the interaction axioms to the base logics, we derive exactly the theorems for the language $\mathcal{L}_{1}$ obtained by only adding axiom $\times$ to $L_{1}$, and nothing else. For the case of conservative characterization, by adding these interaction axioms $\Gamma$, we do not even obtain new theorems for the language $\mathcal{L}_{2}$ as 'side effects', the theorems for this language just remain the same as before.

Finally, we define a notion of minimality among the sets of interaction axioms characterizing an axiom $\mathrm{x}$.

Definition 3. The axiom $x$ is minimally characterized by the set of interaction axioms $\Gamma$ w.r.t. $\left(L_{1}, L_{2}\right)$ when $x$ is characterized by $\Gamma$ w.r.t. $\left(L_{1}, L_{2}\right)$ and there is no set of interaction axioms $\Gamma^{\prime}$ such that $\Gamma>_{\mathrm{L}_{1}+\mathrm{L}_{2}} \Gamma^{\prime}$ and $\mathrm{x}$ is still characterized by $\Gamma^{\prime}$ w.r.t. $\left(\mathrm{L}_{1}, \mathrm{~L}_{2}\right)$.

\subsection{Definability of Modalities and Characterization of Ax- ioms}

The definability of modalities in terms of other modalities is studied from a theoretical point of view by Halpern et al. [17]. This study is subsequently applied to epistemic logic by the same authors in [16]. Three notions of definability emerge from this work: explicit definability, implicit definability and reducibility. It has been proven that, for modal logic, explicit definability coincides with the conjunction of implicit definability and reducibility (unlike first-order logic, where the notion of explicit definability coincides with implicit definability only). In this article, we are interested only in the notion of explicit definability, which is also used in [25]. 
Definition 4. Let $\{i, j\}=\{1,2\}$. The modality $\langle i\rangle$ is explicitly defined in the logic $\mathrm{L}_{i, j}$ in terms of the modality $\langle j\rangle$ by a formula $\operatorname{def}_{i}(p) \in \mathcal{L}_{j}$ if, and only if,

$$
\langle i\rangle p \leftrightarrow \operatorname{def}_{i}(p) \in \mathrm{L}_{i, j} .
$$

The following key theorem will play an important role in the last section. It provides necessary and sufficient conditions for an axiom to be (conservatively) characterized by a set of interaction axioms. It also states that in case one of the modalities is definable in terms of the other, then this characterization is actually minimal (in the sense of Definition 3).

Theorem 1. Assume that $\langle 2\rangle$ is explicitly defined in $L_{1}+L_{2}+\Gamma$ in terms of $\langle 1\rangle$ by a formula $\operatorname{def}_{2}(p) \in \mathcal{L}_{1}$ positive in $p$. Then, the following are equivalent:

- $x$ is characterized by $\Gamma$ w.r.t. $\left(L_{1}, L_{2}\right)$;

- $L_{1}+L_{2}+\Gamma=L_{1}+x+\left\{\langle 2\rangle p \leftrightarrow \operatorname{def}_{2}(p)\right\}$.

Moreover, assume that $\langle 1\rangle$ is also explicitly defined in $L_{1}+L_{2}+\Gamma$ in terms of $\langle 2\rangle$ by a formula $\operatorname{def}_{1}(p) \in \mathcal{L}_{2}$ positive in $p$. Then, the following are equivalent:

- $x$ is conservatively characterized by $\Gamma$ w.r.t. $\left(L_{1}, L_{2}\right)$;

- $L_{1}+L_{2}+\Gamma=L_{1}+x+\left\{\langle 2\rangle p \leftrightarrow \operatorname{def}_{2}(p)\right\}$ and $L_{1}+L_{2}+\Gamma=L_{2}+\left\{\langle 1\rangle p \leftrightarrow \operatorname{def}_{1}(p)\right\}$.

Finally, in both cases, the axiom $\mathrm{x}$ is (conservatively) characterized by $\Gamma$ w.r.t. $\left(L_{1}, L_{2}\right)$ if, and only if, it is minimally (conservatively) characterized by $\Gamma$ w.r.t. $\left(L_{1}, L_{2}\right)$.

\section{$3 \quad$ Epistemic and Doxastic Logics}

In this section, we will see the standard formal semantics of knowledge, belief and conditional belief. For examples and applications of these semantics in computer science, the interested reader can consult [10] or [27]. We will also introduce the convoluted axioms .2, .3 and .4 together with the classes of frames they define.

\subsection{Logics of Knowledge and Belief}

\subsubsection{Epistemic and doxastic languages}

We define the epistemic-doxastic language $\mathcal{L}_{K B}$ by the following BNF grammar:

$$
\mathcal{L}_{K B}: \varphi \quad:=p|\neg \varphi|(\varphi \wedge \varphi)|B \varphi| K \varphi
$$

where $p$ ranges over $\mathbb{P}$. The propositional language $\mathcal{L}_{0}$ is the language $\mathcal{L}_{K B}$ without the knowledge and belief operators $K$ and $B$. The language $\mathcal{L}_{K}$ is the language $\mathcal{L}_{K B}$ without the belief operator $B$, and the language $\mathcal{L}_{B}$ is the 
language $\mathcal{L}_{K B}$ without the knowledge operator $K$. The formula $B \varphi$ reads as 'the agent believes $\varphi$ ' and $K \varphi$ reads as 'the agent knows $\varphi$ '. Their dual operators $\langle B\rangle \varphi$ and $\langle K\rangle \varphi$ are abbreviations of $\neg B \neg \varphi$ and $\neg K \neg \varphi$ respectively.

Remark 1 . We have to be careful with the notion of belief, since the term 'belief' has different meanings: my belief that it will rain tomorrow is intuitively different from my belief that the Fermat-Wilson theorem is correct. This intuitive semantic difference that anyone can perceive stems from the fact that the doxastic strength of these two beliefs are not on the same 'scale'. Lenzen argues that there are two different kinds of belief, which he calls weak and strong belief (or conviction) [24]. A relatively detailed analysis distinguishing weak from strong belief is presented by Shoham and Leyton-Brown [29, p. 414-415]. Also see $[24,3]$. In this paper, we only deal with the notion of strong belief (or conviction) and $B \varphi$ stands for this notion. We will sometimes denote $B_{w}$ for the notion of weak belief.

\subsubsection{Semantics}

In epistemic logic, a semantics of the modal operators of belief $(B)$ and knowledge $(K)$ is often provided by means of a Kripke semantics. The first logical framework combining these two operators with a Kripke semantics was proposed by Kraus and Lehmann [19].

An epistemic-doxastic model $\mathcal{M}$ is a (bi-modal) Kripke model as defined in Section 2.1 where $R_{1}$ is interpreted as the accessibility relation for knowledge and $R_{2}$ is interpreted as the accessibility relation for belief. The truth conditions for $\mathcal{M}, w \models \varphi$ are then defined as in Section 2.1. An epistemic-doxastic frame $\mathcal{F}$ is an epistemic-doxastic model without a valuation. We often denote $R_{K}(w)=$ $\left\{v \in W \mid w R_{K} v\right\}$ and $R_{B}(w)=\left\{v \in W \mid w R_{B} v\right\}$.

\subsubsection{Logics}

Below, we give a list of properties of the accessibility relations $R_{B}$ and $R_{K}$ that will be used in the rest of the article. We also give, below each property, the axiom which defines the class of epistemic-doxastic frames that fulfill this property (see [7, Def. 3.2] for a definition of the notion of definability). We choose, without any particular reason, to use the knowledge modality to write these conditions.

- serial: $R_{K}(w) \neq \emptyset$

Axiom D: $K \varphi \rightarrow\langle K\rangle \varphi$;

- transitive: If $w^{\prime} \in R_{K}(w)$ and $w^{\prime \prime} \in R_{K}\left(w^{\prime}\right)$, then $w^{\prime \prime} \in R_{K}(w)$ Axiom 4: $K \varphi \rightarrow K K \varphi$;

- Euclidean: If $w^{\prime} \in R_{K}(w)$ and $w^{\prime \prime} \in R_{K}(w)$, then $w^{\prime} \in R_{K}\left(w^{\prime \prime}\right)$ Axiom 5: $\neg K \varphi \rightarrow K \neg K \varphi$;

- reflexive: $w \in R_{K}(w)$

Axiom T: $K \varphi \rightarrow \varphi$; 
- symetric: If $w^{\prime} \in R_{K}(w)$, then $w \in R_{K}\left(w^{\prime}\right)$

Axiom B: $\varphi \rightarrow K \neg K \neg \varphi$;

- confluent: If $w^{\prime} \in R_{K}(w)$ and $w^{\prime \prime} \in R_{K}(w)$, then there is $v$ such that $v \in R_{K}\left(w^{\prime}\right)$ and $v \in R_{K}\left(w^{\prime \prime}\right)$

Axiom .2: $\langle K\rangle K \varphi \rightarrow K\langle K\rangle \varphi$;

- weakly connected: If $w^{\prime} \in R_{K}(w)$ and $w^{\prime \prime} \in R_{K}(w)$, then $w^{\prime}=w^{\prime \prime}$ or $w^{\prime} \in R_{K}\left(w^{\prime \prime}\right)$ or $w^{\prime \prime} \in R_{K}\left(w^{\prime}\right)$

Axiom .3: $\langle K\rangle \varphi \wedge\langle K\rangle \psi \rightarrow\langle K\rangle(\varphi \wedge \psi) \vee\langle K\rangle(\psi \wedge\langle K\rangle \varphi) \vee\langle K\rangle(\varphi \wedge\langle K\rangle \psi)$;

- R1: If $w^{\prime} \in R_{K}(w)$ and $w \neq w^{\prime}$ and $w^{\prime \prime} \in R_{K}(w)$, then $w^{\prime} \in R_{K}\left(w^{\prime \prime}\right)$

Axiom .4: $(\varphi \wedge\langle K\rangle K \varphi) \rightarrow K \varphi$.

The logic $\mathrm{KD} 45_{B}$ is the smallest normal modal logic for $\mathcal{L}_{B}$ generated by the set of axioms $\{D, 4,5\}$. For any $x \in\{.2, .3, .4\}$, the logic $S 4 . x_{K}$ is the smallest normal modal logic for $\mathcal{L}_{K}$ generated by the set of axioms $\{\mathrm{T}, 4, \mathrm{x}\}$. We have the following relationship between these logics:

$$
\mathrm{S} 4_{K} \subset \mathrm{S} 4.2_{K} \subset \mathrm{S} 4.3_{K} \subset \mathrm{S} 4.4_{K} \subset \mathrm{S} 5_{K} .
$$

\subsection{Logics of Knowledge and Conditional Belief}

\subsubsection{A language for knowledge and conditional belief}

Taking up the work of Friedman and Halpern [13], we define the syntax of the language $\mathcal{L}_{K B^{q}}$ inductively by the following BNF grammar:

$$
\mathcal{L}_{K B^{q}}: \varphi \quad:=p|\neg \varphi|(\varphi \wedge \varphi)\left|B^{\varphi} \varphi\right| K \varphi
$$

where $p$ ranges over $\mathbb{P}$. The symbol $\top$ is an abbreviation for $p \vee \neg p$, and $B \varphi$ is an abbreviation for $B^{\top} \varphi$. The language $\mathcal{L}_{K}$ is $\mathcal{L}_{K B^{q}}$ without the belief operator $B^{\psi}$, and the language $\mathcal{L}_{B^{\psi}}$ is $\mathcal{L}_{K B^{q}}$ without the knowledge operator $K$.

\subsubsection{Semantics}

Numerous semantics have been proposed for conditional beliefs, such as preferential structures [20], $\epsilon$-semantics [1], possibilistic structures [9], and $\kappa$-ranking $[31,30]$. They all have in common that they validate the axiomatic system $\mathrm{P}$ originally introduced in [20]. This remarkable fact is explained by Friedman and Halpern [13], where a general framework based on plausibility measures is proposed. As proved in that article, plausibility measures generalize all these semantics. So, we adopt the general framework of plausibility measures to provide a semantics for $\mathcal{L}_{K B^{q}}$. Plausibility spaces and epistemic-plausibility spaces were introduced by Friedman and Halpern respectively in [12] and [13]. We can nevertheless mention that other logical formalisms dealing with conditional beliefs are proposed in the economics literature [8]. These other formalisms have been taken up in the field of dynamic epistemic logic $[4,5,6]$. 
If $W$ is a non-empty set of possible worlds, then an algebra over $W$ is a set of subsets of $W$ closed under union and complementation. In the rest of the article, $D$ is a non-empty set partially ordered by a relation $\leq$ (so that $\leq$ is reflexive, transitive and anti-symmetric). We further assume that $D$ contains two special elements $T$ and $\perp$ such that for all $d \in D, \perp \leq d \leq T$. As usual, we define the ordering $<$ by taking $d_{1}<d_{2}$ if and only if $d_{1} \leq d_{2}$ and $d_{1} \neq d_{2}$.

Definition 5. A (qualitative) plausibility space is a tuple $S=(W, \mathcal{A}, P l)$ where:

- $W$ is a non-empty set of possible worlds;

- $\mathcal{A}$ is an algebra over $W$;

- $P l: \mathcal{A} \rightarrow D$ is a function mapping sets of $\mathcal{A}$ into $D$ and satisfying the following conditions:

A0 $P l(W)=\top$ and $P l(\emptyset)=\perp$;

A1 If $A \subseteq B$, then $P l(A) \leq P l(B)$;

A2 If $A, B$, and $C$ are pairwise disjoint sets, $P l(A \cup B)>P l(C)$, and $P l(A \cup C)>P l(B)$, then $P l(A)>P l(B \cup C)$;

A3 If $\operatorname{Pl}(A)=\operatorname{Pl}(B)=\perp$, then $\operatorname{Pl}(A \cup B)=\perp$.

We denote by $\mathcal{S}$ the class of all (qualitative) plausibility spaces.

We can naturally introduce an accessibility relation $R_{K}$ to (qualitative) plausibility space in order to give a semantics to the knowledge modality of the language $\mathcal{L}_{K B^{q}}$. This yields the notion of epistemic plausibility space:

Definition 6. An epistemic-plausibility space is a tuple $\mathcal{M}=\left(W, R_{K}, V, \mathcal{P}\right)$ where:

- $W$ is a non-empty set of possible worlds;

- $R_{K} \in 2^{W \times W}$ is a binary relation over $W$ called an accessibility relation;

- $V: \mathbb{P} \rightarrow 2^{W}$ is a function called a valuation mapping propositional variables to subsets of $W$;

- $\mathcal{P}: W \rightarrow \mathcal{S}$ is a function called a plausibility assignment mapping each world $w \in W$ to a (qualitative) plausibility space $\left(W_{w}, \mathcal{A}_{w}, P l_{w}\right)$ such that $W_{w} \subseteq W$.

We denote by $\mathcal{S}_{K}$ the class of all (qualitative) epistemic-plausibility spaces.

Definition 7. Let $\varphi \in \mathcal{L}_{K B^{q}}$, let $\mathcal{M}$ be an epistemic-plausibility space and let $w \in \mathcal{M}$. The satisfaction relation $\mathcal{M}, w \models \varphi$ is defined inductively as follows:

$$
\begin{array}{lll}
\mathcal{M}, w \models p & \text { iff } & w \in V(p) \\
\mathcal{M}, w \models \varphi \wedge \varphi^{\prime} & \text { iff } & \mathcal{M}, w \models \varphi \text { and } \mathcal{M}, w \models \varphi^{\prime} \\
\mathcal{M}, w \models \neg \varphi & \text { iff } & \text { not } \mathcal{M}, w \models \varphi \\
\mathcal{M}, w \models B^{\psi} \varphi & \text { iff } & \text { either } P l_{w}\left(\llbracket \psi \rrbracket_{w}\right)=\perp \text { or } \\
& & P l_{w}\left(\llbracket \psi \wedge \varphi \rrbracket_{w}\right)>P l_{w}\left(\llbracket \psi \wedge \neg \varphi \rrbracket_{w}\right) \\
\mathcal{M}, w \models K \varphi & \text { iff } & \text { for all } v \in R_{K}(w), \mathcal{M}, v \models \varphi
\end{array}
$$


where $\llbracket \varphi \rrbracket_{w}=\left\{v \in W_{w} \mid \mathcal{M}, v \models \varphi\right\}$. We abusively write $w \in \mathcal{M}$ for $w \in W$, and we also write $\mathcal{M} \models \varphi$ when for all $w \in \mathcal{M}, \mathcal{M}, w \models \varphi$. If $\Gamma$ is a set of formulae (possibly infinite), we write $\mathcal{M} \models \Gamma$ when $\mathcal{M} \models \varphi$ for all $\varphi \in \Gamma$.

\subsubsection{Logics}

The following theorem presents a slightly different version of the axiomatic system P originally introduced by Kraus, Lehmann and Magidor [20] for nonmonotonic logics.

Theorem 2. [13] The following logic $K_{B^{q}}$ is a sound and complete axiomatization of $\mathcal{L}_{B^{q}}$ with respect to all (qualitative) plausibility spaces.

$$
\begin{aligned}
& \text { Prop : All inferences rules of propositional logic and } \\
& \text { substitution instances of propositional tautologies } \\
& C 1: B^{\psi} \psi \\
& C 2:\left(B^{\psi} \varphi_{1} \wedge B^{\psi} \varphi_{2}\right) \rightarrow B^{\psi}\left(\varphi_{1} \wedge \varphi_{2}\right) \\
& \text { C3 : } \quad\left(B^{\psi_{1}} \varphi \wedge B^{\psi_{2}} \varphi\right) \rightarrow B^{\psi_{1} \vee \psi_{2}} \varphi \\
& C 4:\left(B^{\psi} \varphi \wedge B^{\psi} \chi\right) \rightarrow B^{\psi \wedge \varphi} \chi \\
& R C 1 \quad \text { : If } \psi \leftrightarrow \psi^{\prime} \text { then } B^{\psi} \varphi \leftrightarrow B^{\psi^{\prime}} \varphi \\
& R C 2 \text { : If } \varphi \rightarrow \varphi^{\prime} \text { then } B^{\psi} \varphi \rightarrow B^{\psi} \varphi^{\prime}
\end{aligned}
$$

Moreover, $K_{K B^{q}}:=K_{K}+K_{B^{q}}$ is a sound and strongly complete axiomatization for $\mathcal{L}_{K B^{q}}$ with respect to all (qualitative) epistemic-plausibility spaces.

Note that the proof of Theorem 2 in [13] only considers accessibility relations which are equivalence relations (it corresponds to Theorem 11 of [13]). The proof can easily be adapted to our more general setting and the proof shows in fact that the logics are strongly complete. Compactness of $\mathrm{K}_{K B^{q}}$ follows from this strong completeness result.

Corollary 1. $K_{K B^{q}}$ is compact, i.e., for all $\Gamma \subseteq \mathcal{L}_{K B^{q}}$ and all $\varphi \in \mathcal{L}_{K B^{q}}$, if $\Gamma \models \varphi$, then for some finite $\Gamma_{0} \subseteq \Gamma$, we have that $\Gamma_{0} \models \varphi$.

The axiom $\left(B^{q}\left(\varphi \rightarrow \varphi^{\prime}\right) \wedge B^{q} \varphi\right) \rightarrow B^{q} \varphi^{\prime}$ and the inference rule from $\varphi$ infer $B^{q} \varphi$ are both derivable in $\mathrm{K}_{B^{q}}$. Therefore, $\mathrm{K}_{B^{q}}$ is also a normal modal logic. Hence, the language $\mathcal{L}_{K B^{q}}$ can also be given a Kripke semantics: the models would be of the form $\mathcal{M}=\left(W, R_{K},\left\{R_{\psi} \mid \psi \in \mathcal{L}_{K B^{q}}\right\}, V\right)$, where $R_{\psi}$ is an accessibility relation for each $\psi \in \mathcal{L}_{K B^{q}}$. This entails that the first part of Theorem 1 (and its proof) can easily be adapted to the setting of conditional beliefs, where the second modality [2] is replaced by a family of modalities $\left\{B^{q} \mid \psi \in \mathcal{L}_{K B^{q}}\right\}$. We obtain the following theorem:

Theorem 3. Let $\Gamma \subseteq \mathcal{L}_{K B^{q}}$ and $x \in \mathcal{L}_{K B^{q}}$. Assume that for all $\psi \in \mathcal{L}_{K B^{q}}$ the modality $B^{\psi} p$ is explicitly defined in $S 4_{K}+K_{B^{q}}+\Gamma$ in terms of $K$ by a formula $\operatorname{def}_{B^{\psi} p} \in \mathcal{L}_{K}$ positive in $p$. Then, the following are equivalent:

- $x$ is characterized by $\Gamma$ w.r.t. $\left(S 4_{K}, K_{B^{q}}\right)$;

- $S 4_{K}+K_{B^{q}}+\Gamma=S 4_{K}+x+\left\{B^{q} p \leftrightarrow d e f_{B^{q} p}\right\}$. 


\section{Interaction Axioms}

In this section, we will set out the interaction axioms which have been proposed and discussed in the epistemic logic literature and which connect the notions of belief or conditional belief with the notion of knowledge. We will start by reviewing interaction axioms that deal with belief, and then we will consider interaction axioms that deal with conditional belief. Note that a classification of certain interaction principles has been proposed by van der Hoek [33]. ${ }^{2}$

\subsection{Interaction Axioms between Knowledge and Belief}

The following interaction axioms were suggested by Hintikka [18]:

$$
\begin{aligned}
K p & \rightarrow B p \\
B p & \rightarrow K B p
\end{aligned}
$$

Axiom $I_{1}$ is a cornerstone of epistemic logic. Just as axiom $\mathrm{T}$, it follows from the classical analysis of knowledge of Plato presented in the Theaetetus. Axiom $I_{2}$ highlights the fact that the agent has "privileged access" to his doxastic state.

$$
B p \rightarrow B K p
$$

Axiom $I_{3}$ above was suggested by Lenzen [24]. It characterizes a notion of belief corresponding to some sort of conviction or certainty. This kind of belief is therefore different from the notion of weak belief which can be represented by a probability superior to 0.5 , like my belief that "it will rain tomorrow".

Another interaction axiom also introduced by [24] defines belief in terms of knowledge:

$$
B p \leftrightarrow\langle K\rangle K p
$$

Although this definition might seem a bit mysterious at first sight, it actually makes perfect sense, as explained in [24]. Indeed, the left to right direction $B p \rightarrow\langle K\rangle K p$ can be rewritten $K \neg K p \rightarrow \neg B p$, that is, $\neg(K \neg K p \wedge B p)$. This first implication states that the agent cannot, at the same time, know that she does not know a proposition and be certain of this very proposition. The right to left direction $\langle K\rangle K p \rightarrow B p$ can be rewritten $\langle B\rangle \neg p \rightarrow K \neg K p$. This second implication states that, if the agent considers it possible that $p$ might be false, then she knows that she does not know $p$.

The last interaction axiom we will consider is in fact a definition of knowledge in terms of belief:

$$
K p \leftrightarrow(p \wedge B p)
$$

This list of interaction axioms is incomplete. See [3] for more information about interaction axioms and axioms of epistemic logic.

\footnotetext{
${ }^{2}$ The classification is as follows. If $X, Y, Z$ are epistemic operators, $X \varphi \rightarrow Y Z \varphi$ are called positive introspection formulas, $\neg X \varphi \rightarrow Y \neg Z \varphi$ are called negative introspection formulas, $X Y \varphi \rightarrow Z \varphi$ are called positive extraspection formulas, $X \neg Y \rightarrow \neg Z \varphi$ are called negative extraspection formulas, and $X(Y \varphi \rightarrow \varphi)$ are called trust formulas.
} 
Proposition 4. The sets $\left\{I_{1}\right\},\left\{I_{2}\right\},\left\{I_{3}\right\},\left\{I_{4}\right\},\left\{I_{5}\right\}$ and $\left\{I_{1}, I_{2}, I_{3}\right\}$ are sets of interaction axioms with respect to $\left(S 4_{K}, K D 45_{B}\right){ }^{3}$

The collapse of knowledge and belief. In any logic of knowledge and belief, if we adopt axiom 5 for the notion of knowledge, axiom $D$ for the notion of belief and $I_{1}$ as the only interaction axiom, then we end up with counterintuitive properties. First, as noted by Voorbraak [34], we can derive the theorem $B K p \rightarrow K p .^{4}$ This theorem entails that everything one believes to know is in fact true. As it turns out, these axioms are adopted in the first logical framework combining modalities of knowledge and belief [19]. Moreover, if we add the axiom $I_{3}$, we can also prove that $B p \rightarrow K p$. This theorem collapses the distinction between the notions of knowledge and belief.

A systematic approach has been proposed by van der Hoek to avoid this collapse [33]. He showed, thanks to correspondence theory, that any multimodal logic with both knowledge and belief modalities that includes the set of axioms $\left\{\mathrm{D}, 5, I_{1}, I_{3}\right\}$ entails the theorem $B p \rightarrow K p$. He also showed, however, that for each proper subset of $\left\{\mathrm{D}, 5, I_{1}, I_{3}\right\}$, counter-models can be built which show that none of those sets of axioms entail the collapse of the distinction between knowledge and belief. So we have to drop one principle in $\left\{\mathrm{D}, 5, I_{1}, I_{3}\right\}$. Axioms $\mathrm{D}$ and $I_{3}$ are hardly controversial given our understanding of the notion of strong belief. In this case we have to drop either $I_{1}$ or 5 . Voorbraak proposes to drop axiom $I_{1}$. His notion of knowledge, which he calls objective knowledge, is therefore unusual in so far as it implies that you can know something even if you don't believe it. But, as we have said, he clearly warns that this notion applies to any information-processing device, and not necessarily just to humans. Note that Floridi has similar reservations against axiom $I_{1}[11]$, since his notion of being informed shares similar features with Voorbraak's notion of objective knowledge. Halpern also proposes in [15] to drop $I_{1}$ as a general schema, keeping only those instances of $I_{1}$ where $p$ is propositional. This restriction looks a bit ad hoc at first sight. Dropping axiom 5 seems to be the most reasonable choice in light of the discussion about this axiom in Footnote 1.

By dropping 5, we then only have to investigate the logics between S4 and S5 as possible candidates for a logic of knowledge (S5 excluded), as Lenzen did

\footnotetext{
${ }^{4}$ Here is the proof:

$\begin{array}{lll}1 & K p \rightarrow B p & \text { Axiom } \mathrm{I}_{1} \\ 2 & K \neg K p \rightarrow B \neg K p & I_{1}: \neg K p / p \\ 3 & B p \rightarrow \neg B \neg p & \text { Axiom D } \\ 4 & B \neg p \rightarrow \neg B p & 3, \text { contraposition } \\ 5 & B \neg K p \rightarrow \neg B K p & 4: K p / p \\ 6 & \neg K p \rightarrow K \neg K p & \text { Axiom 5 } \\ 7 & \neg K p \rightarrow B \neg K p & 6,2, \text { Modus Ponens } \\ 8 & \neg K p \rightarrow \neg B K p & 7,5, \text { Modus Ponens } \\ 9 & B K p \rightarrow K p & \text { 8, contraposition. }\end{array}$
}

${ }^{3}$ In the set $\left\{I_{1}, I_{2}, I_{3}\right\}$, we implicitly assume that the sets of propositional letters appearing in $I_{1}, I_{2}$ and $I_{3}$ respectively are disjoint so that we can uniformly and independently replace each of them by arbitrary formulas to check whether Expression (1) of Definition 1 holds. 
in $[25]$.

\subsection{Interaction Axioms between Knowledge and Condi- tional Belief}

The following axioms $I_{1}^{q}, I_{2}^{q}$ and $I_{3}^{q}$ are natural conditional versions of the axioms $I_{1}, I_{2}, I_{3}$ : if $q$ is replaced by $\mathrm{T}$, then these three axioms correspond to the axioms $I_{1}, I_{2}, I_{3}$. Axioms $I_{1}^{q}$ and $I_{2}^{q}$ are first introduced by Moses and Shoham [28] and are also adopted by Friedman and Halpern [12]. Axiom $I_{3}^{q}$ is actually introduced by Lamarre and Shoham [22] in the form $B^{q} p \rightarrow B_{w}^{q} K(q \rightarrow p)$.

$$
\begin{aligned}
K p & \rightarrow B^{q} p \\
B^{q} p & \rightarrow K B^{q} p \\
B^{q} p & \rightarrow B^{q} K(q \rightarrow p)
\end{aligned}
$$

Axiom $I_{1}^{q}$ states that, if the agent knows that $p$, then she also believes that $p$, and so on under any assumption $q$. Note that $I_{1}^{q}$ entails the weaker principle $K p \rightarrow\left(q \rightarrow B^{q} p\right)$, which is connected to the Lehrer and Paxton's definition of knowledge as undefeated true belief [23]. Indeed, this derived principle states that if the agent knows that $p$ (formally $K p$ ), then her belief in $p$ cannot be defeated by any true information $q$ (formally $q \rightarrow B^{q} p$ ). Note that this very principle entails an even weaker variant of $I_{1}^{q}$ introduced by Moses and Shoham [28], namely $K p \rightarrow\left(B^{q} p \vee K \neg q\right)$, i.e. $K p \rightarrow\left(\langle K\rangle q \rightarrow B^{q} p\right)$. Axiom $I_{2}^{q}$ is a straightforward generalization of $I_{2}$. As for $I_{3}^{q}$, it states that, if the agent believes $p$ under the assumption that $q$, then, given this very assumption $q$, she also believes that she knows $p$ conditional on $q$.

The axioms $I_{4}^{q}$ and $I_{5}^{q}$ below are also introduced by Lamarre and Shoham [22]:

$$
\begin{aligned}
& \neg B^{q} p \rightarrow K\left(\langle K\rangle q \rightarrow \neg B^{q} p\right) \\
& \langle K\rangle q \rightarrow \neg B^{q} \perp
\end{aligned}
$$

Axiom $I_{4}^{q}$ is a conditional version of axiom $\neg B p \rightarrow K \neg B p$. It is introduced by Lamarre and Shoham [22] in the form $\neg B^{q} p \rightarrow K\left(K \neg q \vee \neg B^{q} p\right)$. Another possible conditional version of axiom $\neg B p \rightarrow K \neg B p$ could have been $\neg B^{q} p \rightarrow$ $K \neg B^{q} p$, and this axiom is indeed adopted in [28]. However, this simpler axiom ignores the possibility of assumptions $q$ which are known to be false $(K \neg q)$ : in that case, these assumptions $q$ should not be taken into account in the reasoning.

Axiom $I_{5}^{q}$ states that, if $q$ is compatible with everything the agents knows, then her beliefs given this assumption cannot be inconsistent. In particular, if $q$ holds then the agent's doxastic state given this assumption cannot be inconsistent: $q \rightarrow \neg B^{q} \perp$ (because $q \rightarrow\langle K\rangle q$ is valid according to axiom T). Axiom $I_{5}^{q}$ is introduced by Lamarre and Shoham [22] in the equivalent form $\langle K\rangle q \rightarrow\left(B^{q} p \rightarrow \neg B^{q} \neg p\right)$. Together with $I_{1}^{q}$ and system $\mathrm{P}$, it entails that knowledge is definable in terms of conditional belief. This definition of knowledge 
actually coincides with the notion of "safe belief" introduced by Baltag and Smets [6]:

$$
K p:=B^{\neg p} \perp
$$

Proposition 5. The sets $\left\{I_{1}^{q}\right\},\left\{I_{2}^{q}\right\},\left\{I_{3}^{q}\right\},\left\{I_{4}^{q}\right\},\left\{I_{5}^{q}\right\}$ and $\left\{I_{1}^{q}, I_{2}^{q}, I_{3}^{q}, I_{4}^{q}, I_{5}^{q}\right\}$ are sets of interaction axioms with respect to $\left(S 4_{K}, K_{B^{q}}\right) .^{5}$

\section{Definability of Knowledge, Belief and Condi- tional Belief}

Obviously, putting together an epistemic logic and a doxastic logic, for example $\mathrm{S} 4_{K}+\mathrm{KD} 45_{B}$, does not yield a genuine epistemic-doxastic logic since the two concepts will not interact. We need to add interaction axioms. Halpern et al. [16] only consider the interaction axioms $I_{1}$ and $I_{2}$ suggested by Hintikka [18]. We will also add the interaction axiom $I_{3}$, suggested by Lenzen [24].

\subsection{Defining Belief in Terms of Knowledge}

Lenzen is the first to note that the belief modality can be defined in terms of knowledge if we adopt $\left\{I_{1}, I_{2}, I_{3}\right\}$ as interaction axioms:

Theorem 6. [25] The belief modality $B$ is explicitly defined in the logic $L:=$ $S 4_{K}+K D 45_{B}+\left\{I_{1}, I_{2}, I_{3}\right\}$ by the formula def $B_{B p}:=\langle K\rangle K p \in \mathcal{L}_{K}$ :

$$
B p \leftrightarrow\langle K\rangle K p \in L
$$

Consequently, the belief modality $B$ is also defined by (Def B) in any logic containing $L$.

As a consequence of this theorem, the belief modality is also explicitly defined by $B p:=\langle K\rangle K p$ in the logics S4. $\mathrm{x}_{K}+\mathrm{KD}_{4} 5_{B}+\left\{I_{1}, I_{2}, I_{3}\right\}$, where $\mathrm{x}$ ranges over $\{.2, .3, .4\}$. This result is in contrast with Theorem 4.8 of [16], from which it follows that the belief modality cannot be explicitly defined in the logic $\mathrm{S} 4 . \mathrm{x}_{K}+$ $\mathrm{KD} 45_{B}+\left\{I_{1}, I_{2}\right\}$, and for any $\mathrm{x} \in\{.2, .3, .4\}$. We see here that the increase in expressivity due to the addition of the interaction axiom $I_{3}$ plays an important role in bridging the gap between belief and knowledge. Note that the definition (Def B) of belief in terms of knowledge corresponds to the interaction axiom $I_{4}$ of Section 4.1.

\footnotetext{
${ }^{5}$ In the set $\left\{I_{1}^{q}, I_{2}^{q}, I_{3}^{q}, I_{4}^{q}, I_{5}^{q}\right\}$, we implicitly assume that the sets of propositional letters appearing in $I_{1}^{q}, I_{2}^{q}, I_{3}^{q}, I_{4}^{q}$ and $I_{5}^{q}$ respectively are disjoint so that we can uniformly and independently substitute each of them by arbitrary formulas to check whether Expression (1) of Definition 1 holds.
} 


\subsection{Defining Knowledge in Terms of Belief}

Defining knowledge in terms of belief depends on the logic of knowledge that we deal with. As the following proposition shows, knowledge can be defined in terms of belief if the logic of knowledge is $\mathbf{5 4 . 4}$, but not if the logic of knowledge is $\mathrm{S} 4$ and S4.x, where $\mathrm{x}$ ranges over $\{.2, .3\}$.

Theorem 7. [3]

1. The knowledge modality $K$ is explicitly defined in the logic $L .4:=S 4_{.} 4_{K}+$ $K D 45_{B}+\left\{I_{1}, I_{2}, I_{3}\right\}$ by the formula def $\operatorname{dop}_{\mathrm{p}}:=p \wedge B p \in \mathcal{L}_{B}$ :

$$
K p \leftrightarrow p \wedge B p \in L .4
$$

2. The knowledge modality $K$ cannot be explicitly defined in the logics S4. $x_{K}+$ $K D 45_{B}+\left\{I_{1}, I_{2}, I_{3}\right\}$ for any $x \in\{.2, .3\}$.

This result can be contrasted with Theorem 4.1 of [16], from which it follows that the knowledge modality cannot be explicitly defined in the logic $S 4.4_{K}+$ $\mathrm{KD} 45_{B}+\{\mathrm{KB} 1, \mathrm{~KB} 2\}$. Again, the increase in expressivity due to the addition of the interaction axiom $I_{3}$ plays an important role in bridging the gap between belief and knowledge.

\subsection{Defining Conditional Belief in Terms of Knowledge}

The conditions under which conditional belief can be defined in terms of knowledge (and reciprocally) have been less explored in the epistemic logic literature.

Theorem 8. The conditional belief modality $B^{q} p$ is explicitly defined in the logic $L^{q}:=S 4_{K}+K_{B^{q}}+\left\{I_{1}^{q}, I_{2}^{q}, I_{3}^{q}, I_{4}^{q}, I_{5}^{q}\right\}$ by the formula def $B_{B^{q} p}:=\langle K\rangle q \rightarrow$ $\langle K\rangle(q \wedge K(q \rightarrow p)) \in \mathcal{L}_{K}$ :

$$
B^{q} p \leftrightarrow(\langle K\rangle q \rightarrow\langle K\rangle(q \wedge K(q \rightarrow p))) \in L^{q}
$$

Moreover, the following also holds:

$$
S 4_{K}+K_{B^{q}}+\left\{I_{1}^{q}, I_{2}^{q}, I_{3}^{q}, I_{4}^{q}, I_{5}^{q}\right\}=S 4.3_{K}+\left\{B^{q} p \leftrightarrow d e f_{B^{q} p}\right\}
$$

The definition (Def $B^{q} p$ ) of conditional belief in terms of knowledge can be viewed as a generalization of the definition of belief in terms of knowledge of Section 4.1: $B p \leftrightarrow\langle K\rangle K p$, that is $I_{4}$. The intuitive interpretations that we gave for $I_{4}$ can also be generalized to the conditional case. The left to right direction of (Def $\left.B^{q} p\right)$ can be rewritten as follows: $\neg\left(B^{q} p \wedge\langle K\rangle q \wedge K(q \rightarrow\langle K\rangle(q \wedge \neg p))\right)$.

It states that it is impossible that the agent believes $p$ given $q$ while at the same time knowing that if $q$ holds then he may consider possible that $p$ does not hold (assuming that he already considers $q$ possible). This seems a reasonable claim. Reciprocally, the right to left direction of (Def $\left.B^{q} p\right)$ can be rewritten as follows: $\neg B^{q} \neg p \rightarrow\langle K\rangle q \wedge K(q \rightarrow\langle K\rangle(q \wedge p))$. It states that if the agent considers that $p$ might be true given $q$, then the agent considers $q$ (epistemically) possible and knows that if $q$ holds then it is possible that $p$ might also hold. This seems also reasonable. 


\subsection{Defining Knowledge in Terms of Conditional Belief}

From Equation (Def $\left.B^{q} p\right)$, we easily obtain that:

Proposition 9. The knowledge modality Kp is explicitly defined in the logic $L^{q}$ by the formula def $\mathrm{f}_{K p}:=B \neg p \perp \in \mathcal{L}_{B^{q}}$ :

$$
K p \leftrightarrow B \neg p \perp \in L^{q}
$$

Proof. To prove that $K p \rightarrow B^{\neg p} \perp \in \mathrm{L}^{q}$, it suffices to observe that $K p \rightarrow$ $B\urcorner^{p} p \in \mathrm{L}^{q}$ by the axiom $I_{1}^{q}$ and that $B^{p} \neg p$ is also an axiom of $\mathrm{L}^{q}$. Therefore, $K p \rightarrow B^{\neg p}(p \wedge \neg p) \in \mathrm{L}^{q}$ by distributivity of $B^{q}$. So, $K p \rightarrow B \neg p \perp \in \mathrm{L}^{q}$. The proof that $B \neg p \perp \rightarrow K p \in \mathrm{L}^{q}$ follows from the contraposition of axiom $I_{5}^{q}$.

The definition (Def $\mathrm{K}^{\prime}$ ) can be rewritten equivalently as follows: $\langle K\rangle p \leftrightarrow$ $\neg B^{p} \neg \top$. So, in a sense, the intuition underlying this definition is that the epistemic possibility that $p$ holds can be identified with the fact that $p$ is compatible with the beliefs of the agent.

\section{Axioms .2, .3 and .4 as Interaction Axioms}

The results of this final section are obtained by applying Theorem 1 either to the results of Lenzen [25] or to the results obtained in the previous section. The first item of Theorem 10 below somehow makes more explicit the fact that .2 is really characterized by the interaction axioms $\left\{I_{1}, I_{2}, I_{3}\right\}$. Lenzen [25] showed that $\mathrm{S} 4_{K}+\mathrm{KD} 45_{B}+\left\{I_{1}, I_{2}, I_{3}\right\}=\mathrm{S} 4.2_{K}+\{B p \leftrightarrow\langle K\rangle K p\}$. However, this expression alone cannot account for the fact that .2 is really characterized by the interaction axioms $\left\{I_{1}, I_{2}, I_{3}\right\}$. As for the second item of Theorem 10, it shows that assuming that knowledge obeys .4 has the same consequences for the logic of knowledge as assuming that knowledge is S4, belief is KD45, and knowledge is true belief.

Theorem 10. 1. The axiom 2 is characterized w.r.t. the pair $\left(S 4_{K}, K D 45_{B}\right)$ by the set of interaction axioms $\left\{I_{1}, I_{2}, I_{3}\right\}$.

2. The axiom 4 is conservatively characterized w.r.t. the pair $\left(S 4_{K}, K D 45_{B}\right)$ by the interaction axiom $I_{5}$.

Proof. It follows from a direct application of Theorem 1 to the results of [25], namely the fact that $\mathrm{S}_{K}+\mathrm{KD} 45_{B}+\left\{I_{1}, I_{2}, I_{3}\right\}=\mathrm{S} 4.2_{K}+\{B p \leftrightarrow\langle K\rangle K p\}$ (for the first result) and $\mathrm{S}_{K}+\mathrm{KD} 45_{B}+\left\{I_{5}\right\}=\mathrm{KD} 45_{B}+\left\{I_{5}\right\}=\mathrm{S} 4.4_{K}+\{B p \leftrightarrow$ $\langle K\rangle K p\}$ (for the second result). To apply Theorem 1, we remind that $p$ is positive in $\langle K\rangle K p$.

Stalnaker [32] argued that the intuition underlying the logic S4.3 consists in defining knowledge as true belief which cannot be defeated by any true fact. In other words, a fact is known if and only if it is true and it will still be believed after learning any true fact. Our interaction axiom $I_{1}^{q}$ formalizes this intuition. 
Theorem 11. The axiom 3 is characterized w.r.t. the pair $\left(S 4_{K}, K_{B^{q}}\right)$ by the set of interaction axioms $\left\{I_{1}^{q}, I_{2}^{q}, I_{3}^{q}, I_{4}^{q}, I_{5}^{q}\right\}$.

Proof. Theorem 1 can easily be adapted to a setting where the second modality [2] is replaced by a family of modalities of the form $B^{q}$. In that case, the proof of the (adapted) Theorem 1 follows the same reasoning (because of the comments that follow Theorem 2). Then, the proof of our theorem follows from a direct application of (an adaptation to the case of conditional belief of) Theorem 1 to the results of Theorems 8, namely the fact that $\mathrm{S}_{K}+\mathrm{K}_{B^{q}}+\left\{I_{1}^{q}, I_{2}^{q}, I_{3}^{q}, I_{4}^{q}, I_{5}^{q}\right\}=$ $\mathrm{K}_{B^{q}}+\left\{K p \leftrightarrow \operatorname{def}_{K p}\right\}=\mathrm{S} 4.3_{K}+\left\{B^{q} p \leftrightarrow \operatorname{def}_{B^{q} p}\right\}$.

\section{Conclusion}

The second item of Theorem 10 tells us that in the epistemic-doxastic context $\left(\mathrm{S} 4_{K}, \mathrm{KD} 45_{B}\right)$, the axiom .4 characterizes the fact that knowledge is actually defined as true belief (a rather strong assumption for knowledge). Although it was acknowledged by all epistemic logicians that axiom .4 characterized knowledge as true belief, this could never be justified and explained rigorously. We claim that our meta-theory of modal logic fills this conceptual gap. Likewise, still in the context of $\left(\mathrm{S} 4_{K}, \mathrm{KD} 45_{B}\right)$, the first item of Theorem 10 tells us that axiom .2 characterizes the fact that the agent knows his beliefs and disbeliefs and that his beliefs are in fact certainties, convictions, and not simply 'weak' beliefs. Theorem 11 provides similar results with conditional beliefs. Stalnaker [32] claimed that S4.3 is a logic where knowledge is defined as true belief which cannot be defeated by any true fact. A similar definition of knowledge is propounded in epistemology by Lehrer and Paxson [23]. The claim of Stalnaker was based on informal semantic arguments. This claim is supported rigorously in our approach by the fact that the axiom .3 is characterized by the set of interaction axioms $\left\{I_{1}^{q}, I_{2}^{q}, I_{3}^{q}, I_{4}^{q}, I_{5}^{q}\right\}$ in the epistemic-doxastic context $\left(\mathrm{S}_{K}, \mathrm{~K}_{B^{q}}\right)$, where we recall that $I_{1}^{q}$ entails $K p \rightarrow\left(q \rightarrow B^{q} p\right)$ : if the agent knows $p$ (formally $K p$ ) then this belief in $p$ cannot be defeated by any true fact $q$ (formally $\left.q \rightarrow B^{q} p\right)$. Hence, our theory of interaction axioms provides a precise and rigorous justification of Stalnaker's 'informal' claim.

Overall, our theory of characterizing axioms via interaction axioms enables us to carry out a rigorous and fine-grained analysis of the intuitive assumptions underlying the logics of knowledge between S4 and S5. It can give precise reasons for choosing a specific epistemic logic for representing and reasoning about a given situation. In a sense, our theory provides meaningful logical foundations for pursuing rigorous epistemological investigations.

Acknowledgements. I thank a reviewer for her/his detailed comments. 


\section{References}

[1] Ernest Adams. The Logic of Conditionals, volume 86 of Synthese Library. Springer, 1975.

[2] Guillaume Aucher. Axioms .2 and .4 as interaction axioms. In Chitta Baral, Giuseppe De Giacomo, and Thomas Eiter, editors, Principles of Knowledge Representation and Reasoning: Proceedings of the Fourteenth International Conference, KR 2014, Vienna, Austria, July 20-24, 2014. AAAI Press, 2014.

[3] Guillaume Aucher. Interdisciplinary Works in Logic, Epistemology, Psychology and Linguistics, volume 3 of Logic, Argumentation and Reasoning, chapter Principles of Knowledge, Belief and Conditional Belief. Springer, 2014.

[4] Alexandru Baltag and Sonja Smets. Conditional doxastic models: A qualitative approach to dynamic belief revision. Electronic Notes in Theoretical Computer Science, 165:5-21, 2006.

[5] Alexandru Baltag and Sonja Smets. Texts in Logic and Games, volume 4, chapter The Logic of Conditional Doxastic Actions, pages 9-31. Amsterdam University Press, 2008.

[6] Alexandru Baltag and Sonja Smets. Texts in Logic and Games, volume 3, chapter A Qualitative Theory of Dynamic Interactive Belief Revision, pages 9-58. Amsterdam University Press, 2008.

[7] Patrick Blackburn, Maarten de Rijke, and Yde Venema. Modal Logic, volume 53 of Cambridge Tracts in Computer Science. Cambridge University Press, 2001.

[8] Olivier Board. Dynamic interactive epistemology. Games and Economic Behavior, 49:49-80, 2004.

[9] Didier Dubois and Henri Prade. Possibilistic logic, preferential model and related issue. In Proceedings of the 12th International Conference on Artificial Intelligence (IJCAI), pages 419-425. Morgan Kaufman, 1991.

[10] Ronald Fagin, Joseph Halpern, Yoram Moses, and Moshe Vardi. Reasoning about knowledge. MIT Press, 1995.

[11] Luciano Floridi. The logic of being informed. Logique et Analyse, 49(196):433-460, 2006.

[12] Nir Friedman and Joseph Y. Halpern. Modeling belief in dynamic systems, part i: Foundations. Artificial Intelligence, 95(2):257 - 316, 1997.

[13] Nir Friedman and Joseph Y. Halpern. Plausibility measures and default reasoning. Journal of the ACM, 48(4):648-685, 2001. 
[14] DM Gabbay, A Kurucz, F Wolter, and M Zakharyaschev. Multidimensional modal logic: Theory and application, volume 148 of Studies in logic and the foundations of mathematics. Elsevier, 1998.

[15] Joseph Y. Halpern. Should knowledge entail belief? Journal of Philosophical Logic, 25(5):483-494, 1996.

[16] Joseph Y. Halpern, Dov Samet, and Ella Segev. Defining knowledge in terms of belief: the modal logic perspective. The Review of Symbolic Logic, 2:469-487, 2009.

[17] Joseph Y. Halpern, Dov Samet, and Ella Segev. On definability in multimodal logic. The Review of Symbolic Logic, 2:451-468, 2009.

[18] Jaakko Hintikka. Knowledge and Belief, An Introduction to the Logic of the Two Notions. Cornell University Press, Ithaca and London, 1962.

[19] Sarit Kraus and Daniel Lehmann. Knowledge, belief and time. In Laurent Kott, editor, Automata, Languages and Programming, volume 226 of Lecture Notes in Computer Science, pages 186-195. Springer Berlin / Heidelberg, 1986.

[20] Sarit Kraus, Daniel J. Lehmann, and Menachem Magidor. Nonmonotonic reasoning, preferential models and cumulative logics. Artificial Intelligence, 44(1-2):167-207, 1990.

[21] F. von Kutschera. Einführung in die intensional Semantik. W. de Gruyter, Berlin, 1976.

[22] Philippe Lamarre and Yoav Shoham. Knowledge, certainty, belief, and conditionalisation (abbreviated version). In $K R$, pages 415-424, 1994.

[23] Keith Lehrer and Thomas Paxson. Knowledge: Undefeated justified true belief. The Journal of Philosophy, 66:225-237, 1969.

[24] Wolfgang Lenzen. Recent Work in Epistemic Logic. Acta Philosophica Fennica 30. North Holland Publishing Company, 1978.

[25] Wolfgang Lenzen. Epistemologische betractungen zu [S4;S5]. Erkenntnis, 14:33-56, 1979.

[26] Maarten Marx and Yde Venema. Multi-dimensional modal logic. Springer, 1997.

[27] John-Jules Ch. Meyer and Wiebe van der Hoek. Epistemic Logic for AI and Computer Science. Cambridge University Press, Cambridge, 1995.

[28] Yoram Moses and Yoav Shoham. Belief as defeasible knowledge. Artificial intelligence, 64(2):299-321, 1993. 
[29] Yoav Shoham and Kevin Leyton-Brown. Multiagent Systems: Algorithmic, Game-Theoretic, and Logical Foundations. Cambridge University Press, 2009.

[30] Wolfgang Spohn. A general non-probabilistic theory of inductive reasoning. In Ross D. Shachter, Tod S. Levitt, Laveen N. Kanal, and John F. Lemmer, editors, UAI, pages 149-158. North-Holland, 1988.

[31] Wolfgang Spohn. Ordinal conditional functions: A dynamic theory of epistemic states. In W. L. Harper and B. Skyrms, editors, Causation in Decision, Belief Change, and Statistics, volume 2, pages 105-134. reidel, Dordrecht, 1988.

[32] Robert Stalnaker. On logics of knowledge and belief. Philosophical studies, 128:169-199, 2006.

[33] Wiebe van der Hoek. Systems for knowledge and belief. Journal of Logic and Computation, 3(2):173-195, 1993.

[34] Frans Voorbraak. As Far as I know. Epistemic Logic and Uncertainty. PhD thesis, Utrecht University, 1993.

\section{A Proofs of Propositions 4 and 5}

Proposition 4. The sets $\left\{I_{1}\right\},\left\{I_{2}\right\},\left\{I_{3}\right\},\left\{I_{4}\right\},\left\{I_{5}\right\}$ and $\left\{I_{1}, I_{2}, I_{3}\right\}$ are sets of interaction axioms with respect to $\left(S 4_{K}, K D 45_{B}\right) .{ }^{6}$

Proof. First, we prove that $\left\{I_{1}\right\},\left\{I_{2}\right\},\left\{I_{3}\right\},\left\{I_{4}\right\},\left\{I_{5}\right\}$ are sets of interaction axioms. It suffices to prove two things, for each $i \in\{1, \ldots, 5\}$ (because of the soundness and completeness of $\mathrm{S} 4_{K}+\mathrm{KD} 45_{B}$ for the language $\mathcal{L}_{K B}$ with respect to the class of epistemic-doxastic models whose accessibility relation $R_{K}$ is transitive and reflexive and whose accessibility relation $R_{B}$ is serial, transitive and Euclidean):

1. there are two pointed epistemic-doxastic models $\left(\mathcal{M}^{1}, w^{1}\right)$ and $\left(\mathcal{M}^{2}, w^{2}\right)$ such that they are bisimilar for $R_{K}$ but $\mathcal{M}^{1}, w^{1} \models I_{i}$ and $\mathcal{M}^{2}, w^{2} \not \models I_{i}$,

2. there are two other pointed epistemic-doxastic models $\left(\mathcal{M}^{1}, w^{1}\right)$ and $\left(\mathcal{M}^{2}, w^{2}\right)$ such that they are bisimilar for $R_{B}$ but $\mathcal{M}^{1}, w^{1} \models I_{i}$ and $\mathcal{M}^{2}, w^{2} \not \models I_{i}$.

We do so for each axiom $I_{1}, I_{2}, I_{3}, I_{4}$ and $I_{5}$. For each of them and for each of the two subcases, we consider two pointed epistemic-doxastic models $\left(\mathcal{M}^{1}, w^{1}\right)=\left(\left\{w^{1}, v^{1}\right\}, R_{K}^{1}, R_{B}^{1}, V^{1}, w^{1}\right)$ and $\left(\mathcal{M}^{2}, w^{2}\right)=\left(\left\{w^{2}, v^{2}\right\}, R_{K}^{2}, R_{B}^{2}, V^{2}, w^{2}\right)$ such that $V^{1}(p)=\left\{w^{1}\right\}$ and $V^{2}(p)=\left\{w^{2}\right\}$ for all $p \in \mathbb{P}$. For each case, only the definitions of the accessibility relations $R_{K}^{1}, R_{K}^{2}, R_{B}^{1}$ and $R_{B}^{2}$ change. For better readability, we omit the superscripts 1 and 2 for the worlds $w$ and $v$, as they should be clear from context.

\footnotetext{
${ }^{6}$ In the set $\left\{I_{1}, I_{2}, I_{3}\right\}$, we implicitly assume that the sets of propositional letters appearing in $I_{1}, I_{2}$ and $I_{3}$ respectively are disjoint so that we can uniformly and independently replace each of them by arbitrary formulas to check whether Expression (1) of Definition 1 holds.
} 
- $I_{1}: K p \rightarrow B p$.

1. $R_{K}^{1}=R_{K}^{2}=R_{B}^{1}=\{(w, w),(v, v)\}$ and $R_{B}^{2}=\{(w, w),(w, v),(v, w),(v, v)\}$;

2. $R_{K}^{1}=R_{B}^{1}=R_{B}^{2}=\{(w, w),(v, v),(w, v),(v, w)\}$ and $R_{K}^{2}=\{(w, w),(v, v)\}$.

- $I_{2}: B p \rightarrow B K p$.

1. $R_{K}^{1}=\{(w, w),(v, v)\}, R_{B}^{1}=R_{B}^{2}=\{(w, v),(v, v)\}$ and $R_{K}^{2}=\{(w, w),(v, v),(v, w)\}$;

2. $R_{K}^{1}=R_{K}^{2}=\{(w, w),(v, v),(w, v)\}, R_{B}^{1}=\{(w, v),(v, v)\}$ and $R_{B}^{2}=$ $\{(w, w),(v, v)\}$.

- $I_{3}: B p \rightarrow K B p$.

1. $R_{K}^{1}=R_{B}^{1}=R_{B}^{2}=\{(w, w),(v, v)\}$ and $R_{K}^{2}=\{(w, w),(v, v),(w, v)\}$;

2. $R_{K}^{1}=R_{K}^{2}=\{(w, w),(v, v),(w, v)\}, R_{B}^{1}=\{(w, v),(v, v)\}$ and $R_{B}^{2}=$ $\{(w, w),(v, v)\}$.

- $I_{4}: B p \rightarrow\langle K\rangle K p$.

1. $R_{K}^{1}=\{(w, w),(v, v),(w, v)\}, R_{B}^{1}=R_{B}^{2}=\{(w, v),(v, v)\}$ and $R_{K}^{2}=$ $\{(w, w),(v, v)\}$

2. $R_{K}^{1}=R_{K}^{2}=\{(w, w),(v, v)\}, R_{B}^{1}=\{(w, v),(v, v)\}$ and $R_{B}^{2}=\{(w, w),(w, v),(v, w),(v, v)\}$.

- $I_{5}: K p \leftrightarrow(p \wedge B p)$.

1. $R_{K}^{1}=R_{B}^{1}=R_{B}^{2}\{(w, w),(v, v)\}$ and $R_{K}^{2}=\{(w, w),(v, v),(w, v),(v, w)\}$;

2. $R_{K}^{1}=R_{B}^{1}=R_{K}^{2}=\{(w, w),(v, v)\}$ and $R_{B}^{2}=\{(w, w),(v, v),(w, v),(v, w)\}$.

The proof for the set of interaction axioms $\left\{I_{1}, I_{2}, I_{3}\right\}$ follows the same method as above. For that case, we consider the same epistemic-doxastic models as in the case $I_{3}$ :

1. $R_{K}^{1}=R_{B}^{1}=R_{B}^{2}=\{(w, w),(v, v)\}$ and $R_{K}^{2}=\{(w, w),(v, v),(w, v)\}$;

2. $R_{K}^{1}=R_{K}^{2}=\{(w, w),(v, v),(w, v)\}, R_{B}^{1}=\{(w, v),(v, v)\}$ and $R_{B}^{2}=$ $\{(w, w),(v, v)\}$.

Proposition 5. The sets $\left\{I_{1}^{q}\right\},\left\{I_{2}^{q}\right\},\left\{I_{3}^{q}\right\},\left\{I_{4}^{q}\right\},\left\{I_{5}^{q}\right\}$ and $\left\{I_{1}^{q}, I_{2}^{q}, I_{3}^{q}, I_{4}^{q}, I_{5}^{q}\right\}$ are sets of interaction axioms with respect to $\left(S 4_{K}, K_{B^{q}}\right){ }^{7}$

Proof. The sets of interaction axioms $\left\{I_{1}^{q}\right\},\left\{I_{2}^{q}\right\},\left\{I_{3}^{q}\right\}$ and $\left\{I_{5}^{q}\right\}$ are dealt with similarly to the way in which we dealt with the set of interaction axioms $\left\{I_{1}\right\},\left\{I_{2}\right\},\left\{I_{3}\right\}$ and $\left\{I_{5}\right\}$. We only need to replace the accessibility relations $R_{B}$ by plausibility spaces $\left(W_{w}, \mathcal{A}_{w}, P l_{w}\right)$ for each $w \in W$. We do so as follows. For any two worlds $w, v \in W_{i}$, if there is an edge from $w$ to $v$ but not from $v$ to $w$,

\footnotetext{
${ }^{7}$ In the set $\left\{I_{1}^{q}, I_{2}^{q}, I_{3}^{q}, I_{4}^{q}, I_{5}^{q}\right\}$, we implicitly assume that the sets of propositional letters appearing in $I_{1}^{q}, I_{2}^{q}, I_{3}^{q}, I_{4}^{q}$ and $I_{5}^{q}$ respectively are disjoint so that we can uniformly and independently substitute each of them by arbitrary formulas to check whether Expression (1) of Definition 1 holds.
} 
then we set $W_{w}=\{w, v\}, P l_{w}(w)=\perp$ and $P l_{w}(v)=\top$. If there is an edge from $w$ to $v$ and an edge from $v$ to $w$ as well, then we set $W_{w}=\{w, v\}, P l_{w}(w)=\top$ and $P l_{w}(v)=\top$. In the first three cases, the counter-example formula is given by considering $q=\top$ and it corresponds to the same formula as for the set of interaction axioms $\left\{I_{1}\right\},\left\{I_{2}\right\}$ and $\left\{I_{3}\right\}$. For the fourth case corresponding to axiom $I_{5}^{q}$, the counter-example is obtained by considering $q=\neg p$.

The set $\left\{I_{4}^{q}\right\}$ is dealt with using the following epistemic-plausibility models:

1. $W_{w}^{1}=W_{w}^{2}=\{w\}, P l_{w}^{1}(w)=P l_{w}^{2}(w)=\top ; W_{v}^{1}=W_{v}^{2}=\{v\}, P l_{v}^{1}(v)=$ $P l_{v}^{2}(v)=\top ;$ and $R_{K}^{1}=\{(w, w),(v, v)\}$ and $R_{K}^{2}=\{(w, w),(v, v),(w, v),(v, w)\} ;$

2. $W_{w}^{1}=\{w, v\}, P l_{w}(w)=\perp, P l_{w}(v)=\top$ and $W_{v}^{1}=\{v\}, P l_{v}^{1}(v)=\top$; $W_{w}^{2}=\{w\}, W_{v}^{2}=\{v\}$ and $P l_{w}^{2}(w)=\{w\}$ and $P l_{v}^{2}(v)=\{v\}$; and $R_{K}^{1}=$ $R_{K}^{2}=\{(w, w),(v, v),(w, v),(v, w)\}$.

The proof for the set $\left\{I_{1}^{q}, I_{2}^{q}, I_{3}^{q}, I_{4}^{q}, I_{5}^{q}\right\}$ is dealt with by considering the same epistemic-plausibility models as for the set $\left\{I_{4}^{q}\right\}$.

\section{B Proof of Theorem 1}

Note that the proof of this theorem appears in [2] without the following Lemma.

Lemma 12. Let $L$ be a normal modal logic for $\mathcal{L}_{\mathbb{A}}$ and let $\Gamma$ be a set of formulas of $\mathcal{L}_{\mathbb{A}}$. Then,

$$
\Gamma \subseteq L \text { iff }\{(\mathcal{M}, w) \in \mathcal{K} \mid \mathcal{M}, w \models L\} \subseteq\{(\mathcal{M}, w) \in \mathcal{K} \mid \mathcal{M}, w \models \Gamma\}
$$

Proof. The left to right direction is straightforward, so we only prove the right to left direction. By contraposition, assume that $\Gamma \nsubseteq \mathrm{L}$. Then, there is $\varphi \in \Gamma$ such that $\varphi \notin \mathrm{L}$. We are going to show that there is $(\mathcal{M}, w) \in \mathcal{K}$ such that $\mathcal{M}, w \not \models \varphi$ and $\mathcal{M}, w \models \mathrm{L}$, so that the right-hand side of Expression (5) does not hold. Assume towards a contradiction that there is no $(\mathcal{M}, w) \in \mathcal{K}$ such that $\mathcal{M}, w \models \mathrm{L} \cup\{\neg \varphi\}$. Then, by the compactness of $\mathrm{K}$, there are $\varphi_{1}, \ldots, \varphi_{n} \in \mathrm{L}$ such that for all $(\mathcal{M}, w) \in \mathcal{K}, \mathcal{M}, w \models \varphi_{1} \wedge \ldots \wedge \varphi_{n} \rightarrow \varphi$. Then, by completeness of the smallest modal logic K, we have that $\varphi_{1} \wedge \ldots \wedge \varphi_{n} \rightarrow \varphi \in \mathrm{K}$. So, because $\mathrm{K} \subseteq \mathrm{L}$ and $\varphi_{1} \wedge \ldots \wedge \varphi_{n} \in \mathrm{L}$, we have by application of Modus Ponens that $\varphi \in \mathrm{L}$. This contradicts the fact that $\varphi \notin \mathrm{L}$. Therefore, there is $(\mathcal{M}, w) \in \mathcal{K}$ such that $\mathcal{M}, w \models \mathrm{L}$ and $\mathcal{M}, w \not \models \varphi$. This proves our result.

Theorem 1. Assume that $\langle 2\rangle$ is explicitly defined in $L_{1}+L_{2}+\Gamma$ in terms of $\langle 1\rangle$ by a formula $\operatorname{def}_{2}(p) \in \mathcal{L}_{1}$ positive in $p$. Then, the following are equivalent:

- $x$ is characterized by $\Gamma$ w.r.t. $\left(L_{1}, L_{2}\right)$;

- $L_{1}+L_{2}+\Gamma=L_{1}+x+\left\{\langle 2\rangle p \leftrightarrow \operatorname{def}_{2}(p)\right\}$.

Moreover, assume that $\langle 1\rangle$ is also explicitly defined in $L_{1}+L_{2}+\Gamma$ in terms of $\langle 2\rangle$ by a formula $\operatorname{def}_{1}(p) \in \mathcal{L}_{2}$ positive in $p$. Then, the following are equivalent: 
- $x$ is conservatively characterized by $\Gamma$ w.r.t. $\left(L_{1}, L_{2}\right)$;

- $L_{1}+L_{2}+\Gamma=L_{1}+x+\left\{\langle 2\rangle p \leftrightarrow \operatorname{def}_{2}(p)\right\}$ and

$L_{1}+L_{2}+\Gamma=L_{2}+\left\{\langle 1\rangle p \leftrightarrow \operatorname{def}_{1}(p)\right\}$.

Finally, in both cases, the axiom $x$ is (conservatively) characterized by $\Gamma$ w.r.t. $\left(L_{1}, L_{2}\right)$ if, and only if, it is minimally (conservatively) characterized by $\Gamma$ w.r.t. $\left(L_{1}, L_{2}\right)$.

Proof. The proof of the second part of the theorem is similar to the proof of the first part. So, we only prove the first part. Assume that $\mathrm{x}$ is characterized by $\Gamma$ w.r.t. $\left(\mathrm{L}_{1}, \mathrm{~L}_{2}\right)$. Then, $\mathrm{L}_{1}+\mathrm{x}=\left(\mathrm{L}_{1}+\mathrm{L}_{2}+\Gamma\right) \cap \mathcal{L}_{1}$, and therefore $\mathrm{L}_{1}+\mathrm{x} \subseteq \mathrm{L}_{1}+\mathrm{L}_{2}+\Gamma$. Moreover, $\langle 2\rangle p \leftrightarrow \operatorname{def}_{2}(p) \in \mathrm{L}_{1}+\mathrm{L}_{2}+\Gamma$ by assumption. Thus, $\mathrm{L}_{1}+\mathrm{x}+\{\langle 2\rangle p \leftrightarrow$ $\left.\operatorname{def}_{2}(p)\right\} \subseteq \mathrm{L}_{1}+\mathrm{L}_{2}+\Gamma$. Now, we prove the converse inclusion. Assume towards a contradiction that there is $\varphi \in \mathrm{L}_{1}+\mathrm{L}_{2}+\Gamma$ such that $\varphi \notin \mathrm{L}_{1}+\mathrm{x}+\left\{\langle 2\rangle p \leftrightarrow \operatorname{def}_{2}(p)\right\}$. Then, there is $\varphi^{\prime} \in \mathcal{L}_{1}$ such that $\varphi \leftrightarrow \varphi^{\prime} \in \mathrm{L}_{1}+\mathrm{L}_{2}+\Gamma$, because $\langle 2\rangle$ is explicitly definable in terms of $\langle 1\rangle$ in $\mathrm{L}_{1}+\mathrm{L}_{2}+\Gamma$. Then, $\varphi^{\prime} \in\left(\mathrm{L}_{1}+\mathrm{L}_{2}+\Gamma\right) \cap \mathcal{L}_{1}$, i.e., $\varphi^{\prime} \in \mathrm{L}_{1}+\mathrm{x}$. Then, by performing the inverse translation that we followed to obtain $\varphi^{\prime}$ from $\varphi$, we conclude that $\varphi \in \mathrm{L}_{1}+\mathrm{x}+\left\{\langle 2\rangle p \leftrightarrow \operatorname{def}_{2}(p)\right\}$. This is impossible, and therefore $\mathrm{L}_{1}+\mathrm{L}_{2}+\Gamma=\mathrm{L}_{1}+\mathrm{x}+\left\{\langle 2\rangle p \leftrightarrow \operatorname{def}_{2}(p)\right\}$.

Now, assume that $\mathrm{L}_{1}+\mathrm{L}_{2}+\Gamma=\mathrm{L}_{1}+\mathrm{x}+\left\{\langle 2\rangle p \leftrightarrow \operatorname{def}_{2}(p)\right\}$. We are going to prove that $\mathrm{L}_{1}+\mathrm{x}=\left(\mathrm{L}_{1}+\mathrm{L}_{2}+\Gamma\right) \cap \mathcal{L}_{1}$. The right to left inclusion is immediate, because $\mathrm{x} \in \mathrm{L}_{1}+\mathrm{L}_{2}+\Gamma$ by assumption. Now, we prove that $\left(\mathrm{L}_{1}+\mathrm{L}_{2}+\Gamma\right) \cap \mathcal{L}_{1} \subseteq$ $\mathrm{L}_{1}+\mathrm{x}$. Because of Lemma 12, this amounts to prove that for all Kripke models $\mathcal{M}$, for all $w \in \mathcal{M}$, if $\mathcal{M}, w \models \mathrm{L}_{1}+\mathrm{x}$, then $\mathcal{M}, w \models\left(\mathrm{L}_{1}+\mathrm{L}_{2}+\Gamma\right) \cap \mathcal{L}_{1}$. In order to do so, we are going to build a Kripke model $\left(\mathcal{M}^{\prime}, w^{\prime}\right)$ such that $(\mathcal{M}, w)$ and $\left(\mathcal{M}^{\prime}, w^{\prime}\right)$ are bisimilar w.r.t. the modality $\langle 1\rangle$ and such that $\mathcal{M}^{\prime}, w^{\prime} \models \mathrm{L}_{1}+\mathrm{x}+$ $\left\{\langle 2\rangle p \leftrightarrow \operatorname{def}_{2}(p)\right\}(*)$, that is, $\mathcal{M}^{\prime}, w^{\prime} \models \mathrm{L}_{1}+\mathrm{L}_{2}+\Gamma$ (recall the assumption). This will prove the second inclusion. If $(\mathcal{M}, w)=\left(W, R_{1}, R_{2}, V, w\right)$, then we define the (pointed) Kripke model $\left(\mathcal{M}^{\prime}, w^{\prime}\right):=\left(W, R_{1}, R_{2}^{\prime}, V, w\right)$, where $R_{2}^{\prime}$ is defined as follows. First, we define the (pointed) Kripke model $\left(\mathcal{M}^{\prime \prime}, w\right):=$ $\left(W, R_{1}, R_{2}, V^{\prime \prime}, w\right)$ by setting $V^{\prime \prime}$ such that for all $q \neq p, V^{\prime \prime}(q)=V(q)$ and such that $V^{\prime \prime}(p)=\{v\}$. Then, for all $u, v \in W$, we set $u R_{2}^{\prime} v$ in $\mathcal{M}^{\prime}$ if, and only if, $\mathcal{M}^{\prime \prime}, u \models \operatorname{def}_{2}(p)$. Then, using the fact that $\operatorname{def}_{2}(p)$ is positive in $p$, one can easily show that $(*)$ holds. This proves the second inclusion.

Finally, we prove the last part of the theorem. Assume towards a contradiction that $x$ is characterized by the set of interaction axioms $\Gamma$ w.r.t. $\left(L_{1}, L_{2}\right)$ and that there is a set of interaction axioms $\Gamma^{\prime}$ such that $\Gamma>_{\mathrm{L}_{1}+\mathrm{L}_{2}} \Gamma^{\prime}$ and such that $x$ is also characterized by $\Gamma^{\prime}$ w.r.t. $\left(\mathrm{L}_{1}, \mathrm{~L}_{2}\right)$. Because $\Gamma>_{\mathrm{L}_{1}+\mathrm{L}_{2}} \Gamma^{\prime}$, we should have that $\mathrm{L}_{1}+\mathrm{L}_{2}+\Gamma \subset \mathrm{L}_{1}+\mathrm{L}_{2}+\Gamma^{\prime}$. However, since $\mathrm{x}$ is characterized by $\Gamma$ and $\Gamma^{\prime}$, we should also have that $\mathrm{L}_{1}+\mathrm{L}_{2}+\Gamma=\mathrm{L}_{1}+\mathrm{L}_{2}+\Gamma^{\prime}=\mathrm{L}_{1}+\mathrm{x}+\left\{\langle 2\rangle p \leftrightarrow \operatorname{def}_{2}(p)\right\}$ by the result of the first part of the theorem. This is impossible.

\section{Proof of Theorem 8}

We first prove a series of lemmata. We also introduce a specific definition of epistemic plausibility space. 
Lemma 13. Let $L$ be a modal logic for $\mathcal{L}_{K B^{q}}$ such that $K_{K B^{q}} \subseteq L$ and let $\Gamma \subseteq \mathcal{L}_{K B^{q}}$. Then,

$$
\Gamma \subseteq L \text { iff }\left\{(\mathcal{M}, w) \in \mathcal{S}_{K} \mid \mathcal{M}, w \models L\right\} \subseteq\left\{(\mathcal{M}, w) \in \mathcal{S}_{K} \mid \mathcal{M}, w \models \Gamma\right\}
$$

Proof. The proof is the same as the proof of Lemma 12, except that we use the completeness and the compactness of $\mathrm{K}_{K B^{q}}$ instead of $\mathrm{K}$, which was proved in Theorem 2 and Corollary 1 respectively.

Definition 8. Let $(\mathcal{M}, w)=\left(W, R_{K}, V, \mathcal{P}\right)$ be a pointed epistemic-plausibility space. The pointed epistemic-plausibility space denoted by $\left(\mathcal{M}^{*}, w^{*}\right)=\left(W^{*}, R_{K}^{*}, V^{*}, \mathcal{P}^{*}, w^{*}\right)$ is defined as follows:

- $W^{*}=W ; R_{K}^{*}=R_{K} ; V^{*}=V$;

- for all $w \in W^{*}, \mathcal{P}^{*}(w)=\left(W_{w}^{*}, \mathcal{A}_{w}^{*}, P l_{w}^{*}\right)$ where:

$-W_{w}^{*}=R_{K}(w)$;

$-\mathcal{A}_{w}^{*}=\mathcal{A}_{w} \cap\left\{R_{K}(w)\right\}$

- $P l_{w}^{*}$ is such that

1. for all $A, B \in \mathcal{A}_{w}^{*}, P l_{w}^{*}(A) \geq P l_{w}^{*}(B)$ iff there is $v \in A$ such that for all $u \in B, v \in R_{K}(u)$;

2. $P l_{w}^{*}(A)=\perp$ iff $A \cap W_{w}^{*}=\emptyset$.

Lemma 14. Let $\mathcal{M}$ be a pointed epistemic-plausibility space such that $\mathcal{M} \models$ S4. $3_{K}$. Then, for all $w \in \mathcal{M}, P l_{w}^{*}\left(\llbracket \psi \wedge \varphi \rrbracket_{w}\right) \geq P l_{w}^{*}\left(\llbracket \psi \wedge \neg \varphi \rrbracket_{w}\right)$ iff $\mathcal{M}, w \models$ $\langle K\rangle(\psi \wedge K(\psi \rightarrow \varphi))$.

Proof. $P l_{w}^{*}\left(\llbracket \psi \wedge \varphi \rrbracket_{w}\right) \geq P l_{w}^{*}\left(\llbracket \psi \wedge \neg \varphi \rrbracket_{w}\right)$

iff there is $v \in R_{K}(w)$ such that $\mathcal{M}, v \models \psi \wedge \varphi$ and for all $u \in R_{K}(w)$ such that $\mathcal{M}, u \mid=\psi \wedge \neg \varphi$, we have that $v \in R_{K}(u)$;

iff there is $v \in R_{K}(w)$ such that $\mathcal{M}, v \models \psi \wedge \varphi$ and for all $u \in R_{K}(w)$, if $v \notin R_{K}(u)$ then $\mathcal{M}, u \not \models \psi \wedge \neg \varphi$;

iff there is $v \in R_{K}(w)$ such that $\mathcal{M}, v \models \psi \wedge \varphi$ and for all $u \in R_{K}(w)$, if $u \in R_{K}(v)$ then $\mathcal{M}, u \models \psi \rightarrow \varphi$ by weak connectedness of $R_{K}$;

iff there is $v \in R_{K}(w)$ such that $\mathcal{M}, v \models \psi \wedge \varphi$ and for all $u \in R_{K}(v)$, $\mathcal{M}, u \models \psi \rightarrow \varphi$ by transitivity of $R_{K}$;

iff $\mathcal{M}, w=\langle K\rangle((\psi \wedge \varphi) \wedge K(\psi \rightarrow \varphi))$;

iff $\mathcal{M}, w=\langle K\rangle(\psi \wedge K(\psi \rightarrow \varphi))$ by reflexivity of $R_{K}$.

Lemma 15. Let $\mathcal{M}$ be an epistemic-plausibility space such that $\mathcal{M}^{*} \models S 4.3_{K}$. Then, for all $w \in \mathcal{M}^{*}$ such that $\mathcal{M}^{*}, w \models\langle K\rangle \psi, P l_{w}^{*}\left(\llbracket \psi \wedge \varphi \rrbracket_{w}\right) \geq P l_{w}^{*}(\llbracket \psi \wedge \neg \varphi \rrbracket)$ iff $P l_{w}^{*}\left(\llbracket \psi \wedge \varphi \rrbracket_{w}\right)>P l_{w}^{*}(\llbracket \psi \wedge \neg \varphi \rrbracket)$.

Proof. Assume that $P l_{w}^{*}\left(\llbracket \psi \wedge \varphi \rrbracket_{w}\right) \geq P l_{w}^{*}\left(\llbracket \psi \wedge \neg \varphi \rrbracket_{w}\right)$ and assume towards a contradiction that $P l_{w}^{*}\left(\llbracket \psi \wedge \varphi \rrbracket_{w}\right)=P l_{w}^{*}\left(\llbracket \psi \wedge \neg \varphi \rrbracket_{w}\right)$. Then, there are $u, v \in$ $R_{K}^{*}(w)$ such that for all $t \in R_{K}^{*}(w)$, we have that $v \in R_{K}^{*}(t), u \in R_{K}^{*}(t)$, $\mathcal{M}^{*}, u \models \psi \wedge \varphi$ and $\mathcal{M}^{*}, v \models \psi \wedge \neg \varphi(*)$. However, $\mathcal{M}^{*}, w \models\langle K\rangle(\psi \wedge K(\psi \rightarrow \varphi))$ 
because $\mathcal{M}^{*} \models B^{q} p \leftrightarrow(\langle K\rangle q \rightarrow\langle K\rangle(q \wedge K(q \rightarrow p)))$ and $\mathcal{M}^{*}, w \models\langle K\rangle \psi$ by assumption. So, there is $t \in R_{K}^{*}(w)$ such that $\mathcal{M}^{*}, t \models K(\psi \rightarrow \varphi)$. This contradicts $(*)$.

Lemma 16. Let $(\mathcal{M}, w)$ be a pointed epistemic-plausibility model such that $\mathcal{M}, w=S 4.3_{K}+\left\{B^{q} p \leftrightarrow(\langle K\rangle q \rightarrow\langle K\rangle(q \wedge K(q \rightarrow p)))\right\}$. Then, for all $\varphi \in$ $\mathcal{L}_{K B^{q}}$, it holds that $\mathcal{M}, w \models \varphi$ iff $\mathcal{M}^{*}, w^{*} \models \varphi$.

Proof. The proof is by induction on $\varphi$.

- $\varphi=p, \varphi=\varphi \wedge \varphi^{\prime}$ and $\varphi=\neg \psi$ work by definition of $V^{*}$ and by Induction Hypothesis;

- $\varphi=K \psi$ works also by Induction Hypothesis because $R_{K}=R_{K}^{*}$;

- $\varphi=B^{\psi} \varphi$.

$\mathcal{M}^{*}, w^{*}=B^{\psi} \varphi$

iff $P l_{w}^{*}\left(\llbracket \psi \rrbracket_{w}\right)=\perp$ or $P l_{w}^{*}\left(\llbracket \psi \wedge \varphi \rrbracket_{w}\right)>P l_{w}^{*}\left(\llbracket \psi \wedge \neg \varphi \rrbracket_{w}\right)$

iff $\llbracket \psi \rrbracket_{w} \cap R_{K}(w)=\emptyset$ or $P l_{w}^{*}\left(\llbracket \psi \wedge \varphi \rrbracket_{w}\right)>P l_{w}^{*}\left(\llbracket \psi \wedge \neg \varphi \rrbracket_{w}\right)$ by Definition 8 , item 2;

iff $\mathcal{M}, w \models K \neg \psi$ or $P l_{w}^{*}\left(\llbracket \psi \wedge \varphi \rrbracket_{w}\right)>P l_{w}^{*}(\llbracket \psi \wedge \neg \varphi \rrbracket)$;

iff $\mathcal{M}, w \models K \neg \psi$ or $P l_{w}^{*}(\llbracket \psi \wedge \varphi \rrbracket w) \geq P l^{*}\left(\llbracket \psi \wedge \neg \varphi \rrbracket_{w}\right)$ by Fact 14 .

iff $\mathcal{M}, w \models K \neg \psi$ or $\mathcal{M}, w \models\langle K\rangle(\psi \wedge K(\psi \rightarrow \varphi))$;

iff $\mathcal{M}, w \models\langle K\rangle \psi \rightarrow\langle K\rangle(\psi \wedge K(\psi \rightarrow \varphi))$ by Fact 15 ;

iff $\mathcal{M}, w \models B^{\psi} \varphi$ by assumption on $\mathcal{M}$.

Theorem 8. The conditional belief modality $B^{q} p$ is explicitly defined in the logic $L^{q}:=S 4_{K}+K_{B^{q}}+\left\{I_{1}^{q}, I_{2}^{q}, I_{3}^{q}, I_{4}^{q}, I_{5}^{q}\right\}$ by the formula def $\operatorname{B}_{B^{q} p}:=\langle K\rangle q \rightarrow$ $\langle K\rangle(q \wedge K(q \rightarrow p)) \in \mathcal{L}_{K}$ :

$$
B^{q} p \leftrightarrow(\langle K\rangle q \rightarrow\langle K\rangle(q \wedge K(q \rightarrow p))) \in L^{q}
$$

Moreover, the following also holds:

$$
S 4_{K}+K_{B^{q}}+\left\{I_{1}^{q}, I_{2}^{q}, I_{3}^{q}, I_{4}^{q}, I_{5}^{q}\right\}=S 4.3_{K}+\left\{B^{q} p \leftrightarrow d e f_{B^{q} p}\right\}
$$

Proof. We split the proof of Expression (Def $B^{q} p$ ) into two parts. First, we prove that $\operatorname{def}_{B^{q} p} \rightarrow B^{q} p \in \mathrm{S}_{K}+\mathrm{K}_{B^{q}}+\left\{I_{1}^{q}, I_{2}^{q}, I_{4}^{q}\right\}$. Second, we prove that $B^{q} p \rightarrow \operatorname{def}_{B^{q}} \in \mathrm{S}_{K}+\mathrm{K}_{B^{q}}+\left\{I_{1}^{q}, I_{3}^{q}, I_{5}^{q}\right\}$.

1. Because of Lemma 13, this amounts to proving that for all pointed epistemic plausibility space $(\mathcal{M}, w)$ such that $\mathcal{M}, w \models \mathrm{S} 4_{K}+\mathrm{K}_{B^{q}}+\left\{I_{1}^{q}, I_{2}^{q}, I_{4}^{q}\right\}$, we have that $\mathcal{M}, w=\operatorname{def}_{B^{q} p} \rightarrow B^{q} p$. Let $(\mathcal{M}, w)$ be such a pointed epistemicplausibility space and assume that $\mathcal{M}, w \mid=\langle K\rangle q \rightarrow\langle K\rangle(q \wedge K(q \rightarrow p))$. Assume towards a contradiction that $\mathcal{M}, w \not \models B^{q} p$. Then, by definition, $P l_{w}\left(\llbracket q \rrbracket_{w}\right) \neq$ $\perp$ and not $P l_{w}\left(\llbracket q \wedge p \rrbracket_{w}\right)>P l_{w}(\llbracket q \wedge \neg p \rrbracket)$. Because $P l_{w}\left(\llbracket q \rrbracket_{w}\right) \neq \perp$, it holds that $\mathcal{M}, w \models \neg B^{q} \perp$. Now, because $\models B^{q} q$ by $\mathrm{C} 1$, we have that $\models B^{q} \neg q \rightarrow B^{q} \perp$ by 
C2, i.e. $\mathcal{M}, w \models \neg B^{q} \perp \rightarrow \neg B^{q} \neg q$. Therefore, $\mathcal{M}, w \models \neg B^{q} \neg q$. So, by axiom $I_{2}^{q}$, $\mathcal{M}, w \mid=\langle K\rangle q$. Then, by assumption, $\mathcal{M}, w \models\langle K\rangle(q \wedge K(q \rightarrow p))$. So, there is $v \in R_{K}(w)$ such that $\mathcal{M}, v \models q \wedge K(q \rightarrow p)$. Therefore, $\mathcal{M}, v \models K(q \rightarrow p)$, and so $\mathcal{M}, v \models B^{q}(q \rightarrow p)$ by application of the rule of necessitation and Axiom $I_{1}^{q}$. Hence, $\mathcal{M}, v \models B^{q} p$, because $\models B^{q} q$. Now, $\mathcal{M}, w \models \neg B^{q} p$, and therefore $\mathcal{M}, w \models K\left(\langle K\rangle q \rightarrow \neg B^{q} p\right)$ by $I_{4}^{q}$. So, $\mathcal{M}, v \models\langle K\rangle q \rightarrow \neg B^{q} p$. Since, $\mathcal{M}, v \models q$, we also have that $\mathcal{M}, v \models\langle K\rangle q$. Therefore, $\mathcal{M}, w \models \neg B^{q} p$ which contradicts our previous deduction. So, we reach a contradiction and therefore our initial assumption was wrong. Hence, we have that $\mathcal{M}, w \models \operatorname{def}_{B^{q}} \rightarrow B^{q} p$. This holds for any pointed epistemic-plausibility space $(\mathcal{M}, w)$ such that $\mathcal{M}, w \models$ $\mathrm{S}_{K}+\mathrm{K}_{B^{q}}+\left\{I_{1}^{q}, I_{2}^{q}, I_{4}^{q}\right\}$, so we have proved the first part.

2. Let $(\mathcal{M}, w)$ be a pointed epistemic-plausibility space. Assume that $\mathcal{M}, w \models \mathrm{S}_{K}+\mathrm{K}_{B^{q}}+\left\{I_{1}^{q}, I_{3}^{q}, I_{5}^{q}\right\}$ and that $\mathcal{M}, w \models B^{q} p$. Assume towards a contradiction that $\mathcal{M}, w \not \models \operatorname{def}_{B^{q} p}$, that is, $\mathcal{M}, w=\langle K\rangle q \wedge K(q \rightarrow\langle K\rangle(q \wedge \neg p))$. Then, $\mathcal{M}, w \models B^{q}(q \rightarrow\langle K\rangle(q \wedge \neg p))$ by $I_{1}^{q}$. So, $\mathcal{M}, w \models B^{q}\langle K\rangle(q \wedge \neg p)$ because $\mathcal{M}, w \models B^{q} q$ and by distributivity of $B^{q}$. Therefore, $\mathcal{M}, w \models B^{q} p \wedge$ $B^{q}\langle K\rangle(q \wedge \neg p)$ because by assumption $\mathcal{M}, w \models B^{q} p$. So, $\mathcal{M}, w \models B^{q} K(q \rightarrow$ $p) \wedge B^{q}\langle K\rangle(q \wedge \neg p)$ by $I_{3}^{q}$. Then, $\mathcal{M}, w \models B^{q}(K(q \rightarrow p) \wedge\langle K\rangle(q \wedge \neg p))$, again by distributivity of $B^{q}$. Therefore, $\mathcal{M}, w \models B^{q}\langle K\rangle(p \wedge \neg p)$. So, $\mathcal{M}, w \models B^{q} \perp$. Thus, by $I_{5}^{q}$, we have that $\mathcal{M}, w \models K \neg q$, that is, $\mathcal{M}, w \models \neg\langle K\rangle q$. This contradicts our assumption that $\mathcal{M}, w \models\langle K\rangle q$. So, we reach a contradiction and therefore our initial assumption was wrong. Hence, we have that $\mathcal{M}, w \models B^{q} p \rightarrow \operatorname{def}_{B^{q}}$. This holds for any pointed epistemic-plausibility space $(\mathcal{M}, w)$ such that $\mathcal{M}, w \models \mathrm{S}_{K}+\mathrm{K}_{B^{q}}+\left\{I_{1}^{q}, I_{3}^{q}, I_{5}^{q}\right\}$, so we have proved the second part by Lemma 13. Putting these two facts altogether, we obtain that $B^{q} p \leftrightarrow \operatorname{def}_{B^{q}} \in \mathrm{S}_{K}+\mathrm{K}_{B^{q}}+\left\{I_{1}^{q}, I_{2}^{q}, I_{3}^{q}, I_{4}^{q}, I_{5}^{q}\right\}$. Hence, we obtain the definability result.

We prove the second part of the theorem. To prove the left to right inclusion of Expression (4), we must show that $\left\{I_{1}^{q}, I_{2}^{q}, I_{3}^{q}, I_{4}^{q}, I_{5}^{q}\right\} \subseteq \mathrm{S} 4.3_{K}+\left\{B^{q} p \leftrightarrow\right.$ $(\langle K\rangle q \rightarrow\langle K\rangle(q \wedge K(q \rightarrow p)))\}$. Because of Lemma 13, this amounts to proving that for all (qualitative) epistemic-plausibility spaces $(\mathcal{M}, w) \in \mathcal{S}_{K}$ such that $\mathcal{M}, w \models \mathrm{S} 4.3_{K}+\left\{B^{q} p \leftrightarrow \operatorname{def}_{B^{q} p}\right\}(*)$ we have that $\mathcal{M}, w \models\left\{I_{1}^{q}, I_{2}^{q}, I_{3}^{q}, I_{4}^{q}, I_{5}^{q}\right\}$. By Lemma 16, this is equivalent to showing that for all $(\mathcal{M}, w) \in \mathcal{S}_{K}$ such that (*) holds, we have that $\mathcal{M}^{*}, w^{*} \models\left\{I_{1}^{q}, I_{2}^{q}, I_{3}^{q}, I_{4}^{q}, I_{5}^{q}\right\}$. We only deal with the cases $I_{4}^{q}$ and $I_{5}^{q}$, the other cases being rather straightforward by definition of $\mathcal{M}^{*}$. We first prove that for all $(\mathcal{M}, w) \in \mathcal{S}_{K}$ such that $(*)$ holds, we have that $\mathcal{M}^{*}, w^{*} \models I_{4}^{q}$. Assume that $\mathcal{M}^{*}, w^{*}=\neg B^{q} p$. Let $v^{*} \in R_{K}^{*}\left(w^{*}\right)$ and assume that $\mathcal{M}^{*}, v^{*} \models\langle K\rangle q$. Then, we must show that $\mathcal{M}^{*}, v^{*} \models \neg B^{q} p$, i.e. $\mathcal{M}^{*}, v^{*}=\langle K\rangle q \wedge K(q \rightarrow\langle K\rangle(q \wedge \neg p))$ because of $(*)$, the rule of necessitation and Lemma 16. We already know that $\mathcal{M}^{*}, v^{*} \models\langle K\rangle q$. Now, because $\mathcal{M}^{*}, w^{*} \models$ $\neg B^{q} p$, we have that $\mathcal{M}^{*}, w^{*}=\langle K\rangle q \wedge K(q \rightarrow\langle K\rangle(q \wedge \neg p))$, and therefore $\mathcal{M}^{*}, w^{*} \models K(q \rightarrow\langle K\rangle(q \wedge \neg p))$. Hence, $\mathcal{M}^{*}, v^{*} \models K(q \rightarrow\langle K\rangle(q \wedge \neg p))$ because $v^{*} \in R_{K}^{*}\left(w^{*}\right)$. This proves that $\mathcal{M}^{*}, w^{*} \models I_{4}^{q}$. Finally, we prove the last case, namely that for all $(\mathcal{M}, w) \in \mathcal{S}_{K}, \mathcal{M}^{*}, w^{*} \models I_{5}^{q}$. Assume that $\mathcal{M}^{*}, w^{*} \models\langle K\rangle q$. We must prove that $\mathcal{M}^{*}, w^{*} \models \neg B^{q} \perp$. That is, by the truth conditions of $B^{q}$, we 
must prove that it is not the case that $P l_{w}^{*}\left(\llbracket q \wedge \perp \rrbracket_{w}\right)>P l_{w}^{*}\left(\llbracket q \wedge \top \rrbracket_{w}\right)$, because $P l_{w}^{*}\left(\llbracket q \rrbracket_{w}\right) \neq \perp$ since $\mathcal{M}^{*}, w^{*} \models\langle K\rangle q$. But $P l_{w}^{*}\left(\llbracket q \wedge \perp \rrbracket_{w}\right)=P l_{w}^{*}(\emptyset)=\perp$ by Condition 2 of Definition 8 . Because it is impossible that for some $d \in D$ we have that $\perp>d$, we obtain our result. Second, we prove the right to left inclusion of Expression (4). For this, we must show that $3 \in \mathrm{L}^{q}:=\mathrm{S}_{K}+\mathrm{K}_{B^{q}}+$ $\left\{I_{1}^{q}, I_{2}^{q}, I_{3}^{q}, I_{4}^{q}, I_{5}^{q}\right\}$ because we already proved in the first part of the theorem that $B^{q} p \leftrightarrow(\langle K\rangle q \rightarrow\langle K\rangle(q \wedge K(q \rightarrow p))) \in \mathrm{L}^{q}:=\mathrm{S}_{K}+\mathrm{K}_{B^{q}}+\left\{I_{1}^{q}, I_{2}^{q}, I_{3}^{q}, I_{4}^{q}, I_{5}^{q}\right\}$. This result is already proved in [3]. This completes the proof of the theorem. 